\title{
Phosphatidylinositol Acyl Chains Configure TLR-Dependent Priming and Activation of the NLRP3 Inflammasome
}

Claire Hamilton ${ }^{1}$, Gerald Larrouy-Maumus ${ }^{2}$, and Paras K. Anand ${ }^{1,{ }^{*}}$

${ }^{1}$ Department of Infectious Disease, Imperial College London, London, W12 ONN, UK.

${ }^{2} \mathrm{MRC}$ Centre for Molecular Bacteriology and Infection, Department of Life Sciences, Imperial College London, London, SW7 3AZ, UK.

${ }^{*}$ Correspondence should be addressed to:

Paras K. Anand,

Department of Infectious Disease,

Imperial College London

Du Cane Road, The Commonwealth Building,

London, W12 0NN.

Tel: (020) 83832063.

E-mail: paras.anand@imperial.ac.uk

Running Title: Phosphatidyl acyl chains regulate NLRP3 activation.

Keywords: NLRP3, Lipid homeostasis, Inflammasome, TLRs, Fatty acids. 


\section{Abstract}

Lipids are important in establishing cellular homeostasis by conducting varied functions including relay of extracellular signals. Imbalance in lipid homeostasis results in metabolic diseases, and is tightly connected to discrepancies in immune signalling. The phosphorylation status of the lipid second messenger phosphatidylinositol phosphates is implicated in key physiological functions and pathologies. By contrast, little is known as to how phosphatidylinositol (PI) lipid acyl chains contribute to cellular processes. Here, by employing a mass-spectrometry-based method, we show a role for PI acyl group chains in regulating NLRP3 inflammasome activation in cells lacking $A B C$ transporter $A B C B 1$. In response to canonical stimuli, $A b c b 1^{-/}$cells revealed defective priming and activation of the NLRP3 inflammasome owing to blunted TLR-dependent signalling. Cellular lipidomics demonstrated that $A B C$ transporter deficiency shifted the total PI balance such that $A b c b 1^{-/}$cells exhibited reduced ratio of the short-chain to long-chain acyl chain lipids. Changes in PI acyl chain configuration accompanied diminished levels of ganglioside GM1, a marker of cholesterol-rich membrane microdomains, in deficient cells. Strikingly, this was not due to differences in the expression of enzymes that either synthesize PI or are involved in acyl chain remodelling. Our study thus suggests an important role for PI lipid chains in priming and activation of the NLRP3 inflammasome thereby highlighting the metabolic regulation of immune responses. 


\section{Introduction}

Cellular membranes not only provide the necessary compartmentalization to establish a molecular gradient across different organelles, but they also uniquely offer a platform for intracellular signalling, differential transport, and pathogen recognition $(1,2)$. The plasma membrane, in particular, is the first line of defence against extracellular pathogens and provides both a physical barrier and a site for localization of diverse membrane proteins. In response to pathogens and other external cues, distinct cell surface receptors including Tolllike receptors, C-type lectin receptors, and other signalling molecules are activated allowing the cell to respond to changing outer environment $(3,4)$.

The identity of most membranous structures is defined by the functions of the localized protein members. Biochemical characterization together with technological developments in massspectrometry have however indicated equally important roles for membrane lipids (5). Together with proteins, lipids form the structural framework of all membranes providing fluidity and flexibility necessary for the functions of integral membrane proteins, and morphological transformations. Glycerophospholipids, sphingolipids, and sterols constitute the most abundant membrane lipids. Glycerophospholipids and sphingolipids, in turn, contain hundreds of lipid species which vary in fatty chain length, linkage, and saturation $(6,7)$.

Lipid entities in membranes are essential to both transmit and regulate the amplitude of signalling by modulating the conformation, localization, and activity of membrane-localized receptors $(8,9)$. One mechanism by which lipids support intracellular signalling is through the presence of cholesterol-rich plasma membrane subdomains known as lipid rafts (10). Lipopolysaccharide (LPS) binding to TLR4 on immune cells results in relocalization of the receptor to the cholesterol rich subdomains in order to drive downstream inflammatory signalling $(4,11)$. Lipids are also required for proper recruitment of the adaptor proteins. To associate with downstream MyD88 adaptor, TIR-domain containing adaptor protein (TIRAP), also known as MyD88- adapter-like (Mal), needs to bind phosphatidylinositol 4,5-bisphosphate (PIP2) prior to being recruited to the plasma membrane to trigger TLR2 signal transduction 
(12). Thus, lipids define some of the key functions at the plasma membrane to drive immune signalling.

TLR activation in turn regulates the priming of the NLRP3 inflammasome, a multimeric protein complex that is activated in response to several PAMPs and DAMPs $(13,14)$. Signalling by TLRs, in an NF-kB-dependent manner, not only upregulates the NLRP3 expression (priming step) but it also results in the synthesis of new mitochondrial DNA (mtDNA) required at the inflammasome activation step to generate oxidized mtDNA released by damaged/dying mitochondria $(15,16)$. Inflammasome assembly, which brings NLRP3 together with procaspase-1 and adaptor molecule ASC, leads to caspase-1 activation which subsequently results in the maturation and release of active forms of cytokines IL-1 $\beta$ and IL-18. The NLRP3 inflammasome plays protective roles during infectious disease but is associated with detrimental functions in inflammatory and autoimmune diseases (17-19).

Immune signalling by TLRs and lipid biogenesis are closely related. TLR activation mostly downregulates lipid synthesis while lipid biogenesis intriguingly is associated with a proinflammatory response (20-22). However, in a mouse model of dyslipidemia, elevated lipid levels resulted in decreased TLR responses (23). Moreover, increased plasma levels of cholesterol and triglycerides lead to reduced secretion of TLR-induced cytokines resulting in enhanced susceptibility of animals to Leishmania infection (23). Other bacterial and viral infections, and metabolic diseases such as diabetes, obesity, lysosomal storage disorders, and atherosclerosis also modify lipid metabolism to calibrate immune responses $(24,25)$.

In response to activating stimuli and other homeostatic mechanisms, cellular lipid metabolism undergoes modifications though the complete landscape of alterations and how distinct changes coordinate immune responses is not fully deciphered. Lipid homeostasis is maintained by the collective action of lipid biosynthesis and trafficking events, regulated by the activity of biosynthetic enzymes and lipid transfer proteins $(26,27)$. Cholesterol homeostasis, on the other hand, is retained by uptake, synthesis, and efflux programs (28, 29). When in excess, the sterol is actively effluxed out by $A B C$ family members, $A B C A 1$ and 
ABCG1 for HDL biogenesis $(30,31)$. Other ABC family members have also been implicated in maintaining membrane lipid composition. For example, ABCB1 (or P-glycoprotein), which has a major role in effluxing chemotoxic drugs and mediating multi-drug resistance (MDR) in several types of malignant cells, transports lipids with broad substrate specificity (32). Importantly, $A B C B 1$ has been suggested to transport a range of lipids at the plasma membrane however the impact on cellular lipid remodelling and subsequent effect on immune signalling by TLRs remains ambiguous.

Here, we demonstrate that modification of the acyl group chains of phosphatidylinositol (PI), a key membrane phospholipid, regulates TLR-dependent signalling in $A b c b 1^{-/}$cells. Cells lacking $A B C$ transporter $A B C B 1$ revealed defective priming and activation of the NLRP3 inflammasome owing to ablated TLR-dependent signalling. Cellular lipidomics by massspectrometry revealed changes in PI fatty acid composition resulting in Pls with long-chain fatty acid moieties in $A b c b 1^{-1}$ cells. Moreover, this shift in $\mathrm{Pl}$ composition accompanied reduced GM1 levels and thus aberrant cholesterol rich membrane microdomains in cells lacking $A B C B 1$. Our study thus suggests an important role for PI lipid chain configuration in the priming of the NLRP3 inflammasome thereby highlighting the metabolic regulation of immune responses.

\section{Results and Discussion}

\section{$A B C$ transporter $B$ family member 1 is required for caspase-1 activation and IL-1 $\beta$ secretion}

Lipid and cholesterol homeostasis within cells are mediated by biosynthesis, efflux, and lipid trafficking events. An excess of cellular lipids could be cytotoxic and is associated with adverse pathological conditions. Members of the ABC family function to exclusively export substrates, mainly lipids and related molecules, out of the cytosol (33). For example, ABCA1 is primarily associated with phospholipid and cholesterol transport to HDL, but it can also flip 
phospholipids such as phosphatidylserine (PS) from the inner to the outer leaflet of the plasma membrane (26) Other $A B C$ members such as $A B C B 1$ have broad substrate specificity in transporting lipids and lipid-related compounds in an ATP-dependent manner (32). Changes in lipid composition as a result of altered $A B C$ transporter activity may affect tissue morphology, function, and immune signalling. One of the key complexes that regulates immune responses during infectious and inflammatory diseases is the NLRP3 inflammasome. While studies have suggested that ABCB1 may alter lipid composition (34), the precise nature of change and the resulting effect on inflammasome activation and immune signalling remains unclear.

In contrast to humans where $\mathrm{ABCB} 1$ has one isoform, two isoforms are expressed in mice, $A b c b 1 a$ and $A b c b 1 b$. We first examined the expression of the two isoforms in bone-marrow derived mouse macrophages (BMDM). PCR amplification followed by gel electrophoresis revealed that both $A b c b 1 a$ and $A b c b 1 b$ are expressed in unstimulated and LPS-stimulated immortalized BMDMs (Fig. S1A and B). However, quantitative PCR revealed that $A b c b 1 b$ expression was approximately 600 -fold higher than that of $A b c b 1 a$, suggesting that this isoform predominates in mouse macrophages (Fig. S1C). Similar results were obtained with primary mouse macrophages (Fig. S1D and data not shown). Additionally, the expression of $A b c b 1 b$ was marginally higher in LPS-stimulated macrophages compared to control cells at all time-points tested, though there was an initial reduction in expression upon LPS stimulation (Fig. S1E). This agrees with previous studies and further suggests a potential role for ABCB1 in immune cells $(35,36)$.

We next used elacridar, a third-generation inhibitor, that has been shown to inhibit ABCB1 activity and overcome drug resistance in cancer models $(37,38)$. To test the activity of elacridar in our cells, we made use of Rhodamine 123 dye. Rhodamine 123 passively diffuses across biological membranes but is subsequently metabolised by intracellular esterases to yield a fluorescent compound which has reduced permeability (39). The efflux of dye out of cells requires active transport by $A B C B 1$ and certain other $A B C$ transporters (39). Primary 
BMDMs treated overnight with increasing concentrations of elacridar and exposed for $30 \mathrm{~min}$ to Rhodamine 123 showed increased sequestration of the dye compared to control cells suggesting inhibition of $A B C B 1$ transporter activity (Fig. 1A). We then examined whether ABCB1 plays a role in NLRP3 inflammasome activation. As before, primary BMDMs were incubated with elacridar overnight to inhibit ABCB1 activity. Subsequently, LPS-primed cells were exposed to nigericin, a potassium ionophore, to activate the canonical NLRP3 inflammasome. NLRP3 inflammasome activation results in the assembly of the inflammasome complex containing NLRP3, the adaptor ASC, and pro-caspase-1. Pro-caspase-1 is then cleaved into its active p20 form, which subsequently results in the maturation and secretion of pro-inflammatory cytokines IL-1 $\beta$ and IL-18. Treatment of macrophages with increasing concentrations of elacridar resulted in a dose-dependent decrease in the generation of cleaved caspase-1 p20 form (Fig. 1B). In addition, this was accompanied with a dosedependent reduction in IL-1 $\beta$ secretion (Fig. 1C). Similar results were observed with the P2X receptor agonist and NLRP3 activator, ATP, revealing reduced caspase-1 cleavage and production of cytokines IL-1 $\beta$ and IL-18 (Fig. S1F, G, Fig. 1D)

While elacridar is known to inhibit $A B C B 1$ function, it has also been reported to inhibit to some extent the function of another $A B C$ transporter, ABCG2 (also referred to as BCRP). Quantitative-PCR demonstrated that $A b c g 2$ mRNA is indeed expressed by mouse macrophages (Fig. S1H). Though, exposure of BMDMs to Ko143, a drug that inhibits ABCG2 exclusively, demonstrated no reduction in caspase-1 cleavage or IL-1 $1 \beta$ secretion following NLRP3 activation (Fig. 1E, F). Together, these data demonstrate the functional requirement of ABCB1 for NLRP3 inflammasome activation.

To validate these results and enable further investigation into the role $A B C B 1$ plays in inflammasome activation, genetic knock-out cell lines were generated using the CRISPR Cas9 approach in immortalised BMDMs. Two cell lines, $A b c b 1 b^{-/} \# 1$ and \#2, were established with deletions in exon 10 of the protein, as verified by Sanger sequencing (Fig. S2A). The mRNA expression of $A b c b 1 b$ in the two CRISPR cell lines was reduced significantly though the PCR 
amplicon was still synthesized to some extent (Fig. S2B). However, compared to WT cells, the protein expression of $A B C B 1$ was diminished by more than $70 \%$ and $90 \%$ in the two cell lines potentially suggesting the presence of unstable mRNA in edited cell lines (Fig. 1G, H). Analysis of $A B C B 1$ transport function showed a significant increase in Rhodamine 123 sequestration in $A b c b 1 b^{-/}$macrophages, confirming reduced efflux of the fluorescent substrate (Fig. 1I) and thus successful $A b c b 1$ deletion in these cells.

We then tested whether NLRP3 inflammasome activation was affected in macrophages with genetic deletion of $A b c b 1 b$. Similar to cells in which ABCB1 had been pharmacologically blocked, $A b c b 1 b^{-/}$macrophages displayed blunted caspase-1 cleavage, in addition to abolished IL-1 $\beta$ secretion compared to WT macrophages during NLRP3 inflammasome activation (Fig. 1J, K). Abcb1 $b^{-/-}$cells exhibited diminished caspase-1 cleavage in LPS-primed cells upon exposure to both nigericin and ATP (Fig. 1J, K). Activation of the NLRP3 inflammasome also leads to an inflammatory cell death, termed pyroptosis, which is mediated by gasdermin-D (GSDMD). Cleavage of GSDMD by caspase-1 results in an $\mathrm{N}$-terminal fragment, which subsequently polymerises and forms pores in the cell membrane, ultimately leading to cell rupture. GSDMD cleavage in $A b c b 1 b^{-/-}$macrophages was diminished in comparison to WT cells following NLRP3 inflammasome activation, further corroborating reduced caspase-1 in deficient cells (Fig. 1J). These data validate a requirement for ABCB1 in NLRP3 inflammasome activation.

\section{$A B C B 1$ is required for TLR-dependent priming and activation of the NLRP3 inflammasome}

NLRP3 inflammasome activation requires two distinct steps. The first, often referred to as the priming step, involves engagement of a TLR and the induction of NF-KB signalling, which in turn leads to the transcriptional and translational upregulation of NLRP3 and pro-IL-1 $\beta$. The second signal assembles and activates the NLRP3 inflammasome complex, leading to 
caspase-1 activation $(17,40)$. We next studied the precise step at which ABCB1 is required for activation of the NLRP3 inflammasome by examining the expression of inflammasome components including NLRP3 and pro-IL-1 $\beta$. Stimulation with LPS and ATP upregulated NLRP3 and pro-IL-1 $\beta$ protein expression in WT cells, which was found to be blunted in cells lacking Abcb1 (Fig. 2A). By contrast, pro-caspase-1 and ASC are constitutively expressed, and their expression remained unaltered in $A b c b 1 b^{-/-}$macrophages (Fig. 1J, 2A). These results coincided with reduced upregulation of NIrp3 and $/ / 1 b$ at the mRNA level (Fig. 2B, C). $\mathrm{NF}-\kappa \mathrm{B}$ is inactive within the cell prior to stimulation through binding to the inhibitory protein IKB. Upon TLR stimulation, signal transduction leads to the activation of the IKK complex, which subsequently phosphorylates $1 \kappa B$, targeting it for degradation. This allows NF- $\kappa B$ to translocate to the nucleus and initiate gene transcription. In addition to NF- $\kappa B$ activation, TLR stimulation also leads to the activation of MAPKs, particularly p38(41). In agreement with above data, the phosphorylation of $1 \kappa B$ and p38 MAPK was reduced in LPS-stimulated $A b c b 1 b^{-/-}$cells compared to WT cells (Fig. 2D). We also examined the expression of other NF$\kappa \mathrm{B}$-dependent cytokines and chemokines in response to TLR4 activation and their expression was similarly decreased in cells lacking ABCB1 (Fig. 2E-G). Moreover, mRNA expression of interferon- $\beta$, which is dependent on adaptor TRIF upon LPS stimulation, was also reduced in $A b c b 1 b^{-/}$cells (Fig. 2H). Furthermore, this response was not specific to TLR4 ligation, as activation of TLR2 or TLR7 by Pam3CSK4 and imiquimod, respectively also resulted in diminished upregulation of NIrp3, $/ 11 \beta$, Tnfa and Cxc/1 (KC) mRNA expression (Fig. 2I, S3). These data suggested blunted TLR -dependent signalling in the absence of ABCB1.

We next tested the requirement of ABCB1 during NLRC4 and AIM2 inflammasomes. Unlike NLRP3, the expression of NLRC4 and AIM2 is constitutively expressed at significant levels in mouse macrophages. However, the expression and therefore the secretion of downstream effector cytokine IL-1 $\beta$ still requires upregulation by TLR signalling. Activation of NLRC4 inflammasome by Salmonella infection and AIM2 inflammasome by exposure to poly(dA:dT) resulted in comparable caspase-1 cleavage between WT and $A b c b 1 b^{-/-}$cells (Fig. S4A and 
data not shown). However, the levels of IL-1 $\beta$ secretion, as expected, were found diminished in both $A b c b 1 b^{-/}$cells and WT cells where ABCB1 was pharmacologically blocked during NLRC4 and AIM2 inflammasome activation (Fig. S4B, C). In contrast to pro-IL-1 $\beta$, pro-IL-18 is constitutively expressed and does not require TLR signalling for upregulation. In agreement, no difference in IL-18 production was observed following NLRC4 or AIM2 inflammasome activation in cells lacking ABCB1 (Fig. S4D). Secretion of IL-18, however, following NLRP3 activation showed complete abolishment in $A b c b 1 b^{-/}$macrophages (Fig. S4D). These data confirm that the NLRC4 and AIM2 inflammasomes are unaffected by Abcb1b-deficiency, and that the diminished IL-1 $\beta$ production is due to reduced TLR- and NF- $\kappa B$-dependent upregulation of pro-IL-1 $\beta$ in $A b c b 1 b^{-/}$cells (Fig. 2C, D).

\section{ABCB1 deficiency disrupts GM1-postive membrane microdomains}

Induction of most signalling cascades at the plasma membrane relies on the presence of highly structured membrane domains. These membrane microdomains, also termed as lipid rafts, are enriched in cholesterol and contribute to the formation of signalling complexes by favouring interaction between molecules $(42,43)$. Cholesterol in membrane microdomains shows higher affinity for sphingolipids, and they together provide structural support to lipid rafts (44). Furthermore, cholesterol can also regulate immune responses independently of its' functions in membrane microdomains. Thus, cellular cholesterol is tightly regulated through intricate uptake, biosynthesis and efflux pathways.

Cellular cholesterol efflux, in particular, is mediated by ABCA1 and ABCG1 which transport excess cholesterol to apoA-I and HDL respectively (30). ABCB1 has also been implicated in cholesterol regulation at the plasma membrane, with studies suggesting that $A B C B 1$ may redistribute the plasma membrane sterol or enhance its' esterification $(45,46)$. However, whether this effect is direct or if ABCB1 alters the cellular lipid landscape is not known. Thus, we next assessed the total levels of cholesterol and any impact on sterol esterification 
qualitatively in WT and $A b c b 1 b^{-/}$macrophages by staining with filipin, a compound that binds to free unesterified cholesterol (25). Excitation of filipin by UV fluorescence showed no obvious differences in staining or distribution of cholesterol between WT and $A b c b 1 b^{-/}$cells. As a control, we exposed WT cells to U18666a, which specifically blocks the lysosomal cholesterol transporter NPC1 (25), and this resulted in expected punctate filipin staining suggestive of lysosomal cholesterol accumulation. (Fig. 3A). Additionally, quantitative analysis of total cellular cholesterol revealed similar levels in both WT and $A b c b 1 b^{-/}$cells (Fig. 3B). These data indicated no major effect of ABCB1 deficiency on cholesterol esterification, distribution, and efflux.

Alternatively, $A B C B 1$ has been suggested to potentially flip cholesterol molecules from the outer leaflet to the inner leaflet (34). Thus, the absence of ABCB1 may potentially disrupt the formation of cholesterol-rich membrane domains. To test this hypothesis, we analysed by flow cytometry the presence of GM1 ganglioside, a glycosphingolipid primarily located in membrane microdomains (47). Cholera toxin subunit B (CTB) selectively binds GM1 ganglioside on the plasma membrane, and is utilised as a probe for GM1 staining (48). Staining of WT cells with Alexa Fluor 488-conjugated CTB showed the presence of GM1 on all cells. However, deletion of ABCB1 reduced GM1 expression in both unstimulated and LPSstimulated cells (Fig. 3C-F). Similar decrease in GM1 expression could be observed when membrane microdomains were disrupted in WT cells by exposure to methyl- $\beta$-cyclodextrin $(\mathrm{M} \beta \mathrm{CD}$ ), a water-soluble compound which alters cholesterol levels in membranes (Fig. 3C, D). These data indicate the disruption of membrane microdomains in $A b c b 1 b$-deficient cells.

The molecules enriched in membrane microdomains are associated with distinct signalling processes. Thus, any changes in membrane microdomains may alter distinct biological processes. As cholesterol is important for the stability and architecture of membrane microdomains, its' composition in membrane microdomains not only influences signalling but also impacts the intake of molecules through diffusion or receptor mediated uptake. Since $M \beta C D$ affects lipid membrane microdomains, we investigated whether disruption of these 
domains will reflect results seen in $A b c b 1 b^{-/-}$macrophages. Disruption of lipid membrane microdomains in WT macrophages with MBCD prior to NLRP3 inflammasome activation led to decreased upregulation of NLRP3 protein expression, while, as expected, the expression of ASC remained unchanged (Figure $\mathbf{3 G}$ ). This coincided with a decreased secretion of IL-1 $\beta$ (Figure $3 \mathrm{H}$ ). Like $A b c b 1 b^{-/-}$cells, disruption of membrane microdomains in WT cells led to a decrease in TNF- $\alpha$ secretion (Figure 3I). Moreover, this was accompanied by a decrease in the mRNA expression of NIrp3, II-1 $\beta$ and Tnf- $\alpha$ suggesting a defect in inflammasome priming (Fig. J-L).

\section{Blunted NLRP3 inflammasome priming and activation is associated with change in PI lipid chains}

A notable feature of the plasma membrane is the distinct lipid composition in the two leaflets of the bilayer (49). Sphingolipids are mostly present in the outer leaflet while glycerophospholipids such as phosphatidylethanolamine (PE), phosphatidylserine (PS), and phosphatidylinositol $(\mathrm{PI})$ are mostly restricted to the inner leaflet thereby imparting distinct functions to the membranes (49). To further investigate the mechanisms by which ABCB1 may be disrupting cholesterol-rich membrane microdomains, whole cell lipidomics was performed by MALTI-TOF MS. Such analysis revealed an altered composition of fatty acid chains of phosphatidylinositol $(\mathrm{PI})$, a lipid associated with membrane microdomains and a precursor for the generation of signalling molecules such as PIP2 and PIP3 (Fig. 4A-C). Two major peaks were observed at $\mathrm{m} / \mathrm{z} 861.4$ and 885.4 , corresponding to $\mathrm{PI}(36: 2)$ and $\mathrm{PI}(38: 4)$ respectively. $A b c b 1 b^{-/-}$cells displayed a higher proportion of $\mathrm{PI}(38: 4)$, indicative of a shift towards PIs with longer chain fatty acids, whereas WT cells had a higher proportion of PI (34:2) containing shorter chain fatty acid moieties (Fig. 4A-C). In addition, lipid analysis by MS also unveiled several ganglioside peaks that were distinct between WT and $A b c b 1 b^{-1-}$ macrophages. Further analysis demonstrated that the total percentage of ganglioside GM1 was reduced in $A b c b 1 b^{-/-}$cells (Fig. 4D) further supporting our GM1 data obtained above by 
flow cytometry (Fig. 3C-F). These data indicate that ABCB1 is essential for maintaining cellular lipid composition, and that deficiency in this transporter affects membrane microdomain formation by altering PI acyl chain composition.

The de novo synthesis of PI occurs at ER through the conversion of phosphatidic acid (PA) and via 2 enzymatic reactions. PA is converted into an intermediate, CDP-DG, by CDP-DG synthetases (CDS), which is subsequently a substrate for CDP-Diacylglycerol--Inositol 3Phosphatidyltransferase (CDIPT, also referred to as phosphatidylinositol synthase) an enzyme that catalyses the conversion of CDP-DG to PI (50). While the acyl chain composition of PA may influence the eventual acyl chain composition of the generated PI, different acyl chains compositions can be achieved through acyl chain remodelling. Cleavage of the fatty acid by phospholipases A, followed by acylation by acyl-CoA-specific lysophospholipid acyltransferase enzymes, is needed to incorporate a new fatty acid molecule (51). Lysophosphatidylinositol acyltransferase (LPIAT) has been shown to reacylate PI with polyunsaturated substrates such as arachidonic acid, while lysocardiolipin acyltransferase 1 (LCLAT1) has been demonstrated to reacylate with steric acid (51). As Abcb1b-deficient macrophages displayed altered fatty acid acyl chains of PI, we investigated whether this was due to disturbances in PI synthesis and acyl chain remodelling. mRNA expression of the acylCoA-specific lysophospholipid acyltransferase enzymes, Lpiat and Lclat1, revealed no significant changes in there expression in $A b c b 1 b^{-/}$macrophages in comparison to WT cells (Figure 4E and F). Similarly, mRNA expression of Cdipt remained unchanged (Figure 4G). These data indicate that ablation of Abcb1 in macrophages disrupts $\mathrm{PI}$ acyl chain composition independently of synthesis or remodelling of its acyl chain composition.

Activation of the NLRP3 inflammasome requires two distinct steps resulting in the upregulation of protein expression for NLRP3 and pro-IL-1 $\beta$, often called the priming step. Following sensing of an appropriate upstream event, the NLRP3 inflammasome complex assembles for activation. Recent reports have however suggested a tight link between the priming and the activation steps of the NLRP3 inflammasome. It has been demonstrated that TLR priming of 
macrophages enables the synthesis of new mitochondrial DNA which is later required to generate oxidized mitochondrial DNA during the inflammasome activation step (15). In the context of our data, this suggests that the PI acyl chain composition may also coordinate both the priming and activation of the NLRP3 inflammasome.

Lipid homeostasis is tightly regulated by the coordination between intake and efflux mechanisms. Cholesterol homeostasis, in particular, is aided by uptake in the form of LDL particles and by efflux to HDL by $A B C$ family members. When limiting levels of extracellular cholesterol are available, de novo cholesterol synthesis is triggered by translocation of ERresident SREBP2 to the nucleus for the expression of genes involved in cholesterol biosynthesis. Intriguingly, both cholesterol and lipid biosynthesis share the same underlying activation mechanisms (20). When in excess, cholesterol efflux is mediated by ABCA1 and $A B C G 1$. While $A B C B 1$ and other $A B C$ transporters are associated with the efflux of lipids and related molecules, their precise substrates remain unknown. Here, we found that deficiency in $A B C B 1$ results in a shift in the balance of total $\mathrm{PI}$ levels resulting in an elevated presence of long-chain acyl chain lipids. A previous study suggested that mutations in p53 gene resulted in Pls containing reduced-length acyl chains (52). PIPs are important signal transducers and relay signalling by changing the phosphorylation status of the inositol sugar head group. Importantly, in the p53 study, while the phosphorylation status of inositol changed, the lipid chains remained unperturbed when cells were grown in the absence of serum (52). This suggests that in PI lipids, the phosphorylation of PIs potentially regulates distinct events to that regulated by the acyl chain composition. Additionally, because of the possible saturation, the carbon content, and the distinct fatty acids that can be added to PI, changes in acyl chain lipids provide much greater plasticity to regulate cellular processes than possible with the seven distinct phosphorylation states of Pls (52). Together, our results suggest an additional layer of metabolic regulation of immunity by PI lipid chains. 


\section{Materials and Methods}

\section{Ethics Statement}

Experiments involving animals were performed in accordance with the Animals (Scientific Procedures) Act 1986, in accordance with a current UK Home Office licence and with approval from the Imperial College Animal Welfare and Ethical Review Body (AWERB).

\section{Bone-marrow derived macrophage isolation and cell culture}

Bone marrow obtained from C57BL/6 mice (Charles River, UK) was isolated from the femurs and tibias of $6-8$ week old mice as previously described $(53,54)$. Bone marrow cells were then incubated in Dulbecco's Modified Eagle Medium (DMEM) containing 10\% heat-inactivated fetal bovine serum (FBS), 1\% penicillin/streptomycin, 1\% HEPES, and 30\% conditioned media from L929 fibroblasts for 5 to 6 days at $37{ }^{\circ} \mathrm{C}$ and $5 \% \mathrm{CO}_{2}$ in order for macrophage

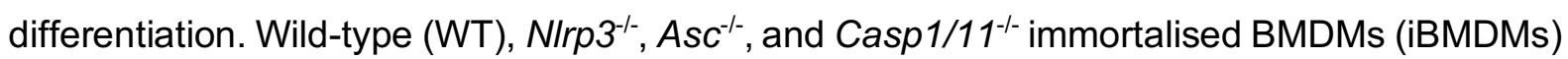
were kindly provided by Katherine Fitzgerald and grown in DMEM containing 10\% FBS, $1 \%$ penicillin/streptomycin, 1\% HEPES, and 10\% L929 conditioned medium. Cells were incubated and grown at $37{ }^{\circ} \mathrm{C}, 5 \% \mathrm{CO}_{2}$ and passaged every 2-3 days. HEK293T cells were grown in DMEM containing $\%$ FBS and $1 \%$ HEPES at $37^{\circ} \mathrm{C}, 5 \% \mathrm{CO}_{2}$. Cells were grown until confluent and passaged every 2-3 days.

\section{Generation of CRISPR-Cas9 ABCB1b knock-out cells}

Guides targeted exon 10 and exon 11 of the $A b c b 1 b$ gene were designed using the CHOPCHOP online software (http://chopchop.cbu.uib.no/) and the Zhang Lab CRISPR design software (http://crispr.mit.edu/). The guides contained a BsmB/ overhang and were as follows: Exon 10 gRNA - CACCGAAGCCTTTGCAAACGCACGA and its reverse complement AAACTCGTGCGTTTGCAAAGGCTTC and exon 11 gRNA CACCGCCCATCGAGAAGCGAAGTTC and its reverse complement AAACGAACTTCGCTTCTCGATGGGC. Guide RNAs were annealed and ligated into the LentiCRISPRv2 plasmid (Addgene \#52961) at the BsmBI restriction site. The recombinant plasmids (LentiCRISPRv2.gRNA10, LentiCRISPRv2.gRNA11) were then transformed into 
competent Escherichia coli (NEB) according to manufacturer's instructions. $1.85 \mu \mathrm{g}$ of LentiCRISPRv2gRNA10 plamsmid or LentiCRISPRv2gRNA10 plasmid, 0.42ug pVSVg plasmid and $1.3 \mu \mathrm{g}$ psPAX2 plasmid were together transfected into the HEK923T cells using polyethylenimine (PEl; Sigma), at a ratio of $1 \mu \mathrm{g}$ DNA: $3 \mu \mathrm{g}$ PEl. Supernatants containing lentiviral particles from transfected HEK293T cells were harvested at 48 hours post transfection. $300 \mu \mathrm{l}$ of lentiviral particle- containing supernatant was then added to the iBMDMs and cells were incubated at $37^{\circ} \mathrm{C}$ for approximately 16 hours after which fresh media was added to cells. Forty-eight hours following transduction, puromycin ( $6 \mu \mathrm{g} / \mathrm{ml}$, Sigma) was added for 7-10 days to select for cells that had been successfully transduced. Puromycinresistant cells were then trypsinised and seeded into a 96-well plate at an approximate concentration of 1 cell per well to obtain clonal cell population. Single cell colonies were identified and expanded followed by Sanger sequencing to identify mutations in Exon 10 or 11 of the $A b c b 1 b$ gene. 2 clones, $A b c b 1 b^{--} \# 1$ and \#2 were identified with deletions of 32 and 9 base pairs in exon 11, respectively (Fig. S2).

\section{Cell stimulations}

Primary and immortalised macrophages were seeded into either 6-well plates at a concentration of $2 \times 10^{6}, 12$ well plates at a concentration of $1 \times 10^{6}$ or 24 -well plates at a concentration of $0.5 \times 10^{6}$ cells per well. Where indicated, macrophages were treated with elacridar (1-10 $\mu \mathrm{M}$, SML0486 Sigma) or Ko143 (50-200 ng/ml, K2144, Sigma) for 16 hours. In experiments with $M \beta C D(C 4555$, Sigma), $M \beta C D(5-10 \mu M)$ was added 30 minutes prior to addition of LPS.

\section{Inflammasome activation}

To activate the NLRP3 inflammasome, cells were incubated with LPS (500 ng/ml, Invivogen) for 4-6 hours to prime the macrophages, followed by ATP $(0.5 \mu \mathrm{M}$, Sigma) or Nigericin (20 $\mathrm{nM}$, Tocris) for approximately 45 minutes. For NLRC4 inflammasome activation, the Salmonella Typhimurium strain SL1344 (kind gift from Jorge Galan and Avinash Shenoy) was cultured overnight in $5 \mathrm{ml}$ Luria-Bertani (LB) broth at $37^{\circ} \mathrm{C}$ and on a shaker at $220 \mathrm{rpm}$. The bacteria were then added to the indicated cells, treated with or without elacridar $(1-5 \mu \mathrm{M})$ or 
$A b c b 1 b^{-/}$cells, at an $\mathrm{MOI}$ of 2 and incubated for approximately 4 hours. For AIM2 inflammasome activation, macrophages were transfected with $1 \mu \mathrm{g}$ of poly(dA:dT) (Invivogen) complexed with Lipofectamine 2000 at a 1:3 ratio according to the manufacturer's instructions for approximately 4-5 hours.

\section{Cell signalling experiments}

The indicated cells were seeded into 6 -well plates at a concentration of $2 \times 10^{6}$ cells per well and incubated with elacridar $(5 \mu \mathrm{M})$ overnight. The following morning media was replaced and cells were stimulated with LPS (500 $\mathrm{ng} / \mathrm{ml})$ for the following time points: 0 (no LPS control), 0.5, 1, 2 and 4 hours. Supernatants were discarded from each well and the cells were subsequently washed with PBS and cells were collected in RIPA buffer. Samples were incubated on ice for approximately 30 minutes before being centrifuged at $15000 \mathrm{xg}$ for 15 minutes at $4^{\circ} \mathrm{C}$ to remove nuclei. The supernatant was collected and the protein concentration of each sample was measured using the BCA Protein Assay kit (Thermo Scientific \#23227) according to manufacturer's instructions. Samples were all standardised to $1 \mu \mathrm{g} / \mu \mathrm{l}$ before immunoblot analysis.

\section{Immunoblot analysis}

For immunoblot of phosphospecific antibodies, cells were collected in RIPA lysis buffer containing both protease and phosphatase inhibitors (Roche) and standardised to $1 \mu \mathrm{g} / \mu \mathrm{l}$ as mentioned above. Samples were boiled at $95{ }^{\circ} \mathrm{C}$ for 5 minutes before being resolved on $12 \%$ SDS-PAGE gels. For immunoblotting of caspase-1, NLRP3, IL-1 $\beta$, ASC, GSDMD and GAPDH, lysates were collected in cell lysis buffer containing NP-40, DTT, and protease inhibitors. Samples were boiled at $95{ }^{\circ} \mathrm{C}$ for approximately $20-30$ minutes before being resolved on $12 \%$ SDS-PAGE gels. SDS-PAGE gels were transferred to nitrocellulose membranes (GE Life Sciences) and subsequently blocked in $5 \%$ milk solution in TBS-Tween $(0.05 \%)$. Membranes were then incubated with the primary antibody overnight at $4{ }^{\circ} \mathrm{C}$ followed by incubation with the HRP-conjugated secondary antibody at room temperature for 1 hour. The primary antibodies used were as follows: P-glycoprotein (1:1000, Invitrogen MA1-2652), Caspase-1 (1:2000, AdipoGen \#AG-20B-0042-C100), NLRP3 (1:2000, AdipoGen \#AG-20B- 
0014-C100), ASC (1:2000, Adipogen \#AG-25B-0006, AL177), GSDMD (1:1000, Abcam \#ab209845), IL-1 $\beta$ (1:500, Cell Signalling \#12426), GAPDH (1:2500, ThermoFisher \# MA5-

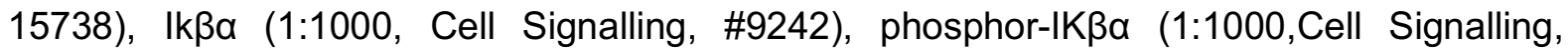
\#2859S), p38 MAPK (1:1000, Cell Signalling, \#9212), phospho-p38 MAKP (1:1000, Cell Signalling, \#4511), AKT (1:1,000; 4691; Cell Signaling Technology), phospho-AKT (1:1,000; 4060; Cell Signaling Technology), p70-S6K1 (1:1,000; 2708; Cell Signaling Technology), phospho-p70-S6K1 (1:1,000; 9234; Cell Signaling Technology). The HRP-conjugated secondary antibodies (ThermoFisher) were used at 1:5000. Following secondary antibody incubation, proteins were visualised using either BioRad Clarity ECL substrate (BioRad \#1705060) or the Pierce ECL Western Blotting Substrate (Thermo Scientific \#32209) and processed on a Bio-Rad imager. Images were obtained using the Bio-Rad software, ImageJ.

\section{ELISA}

Cell culture supernatants were measured for IL-1 (eBioscience \#88-7013-88), TNF- $\alpha$ (eBioscience \#88-7324-88) and IL-18 (MBL \#7625), using ELISA kits according to the manufacturer's instructions.

\section{Real-time PCR}

RNA was isolated using TRIzol (Sigma \# T9424) according to manufacturer's instructions. 250 $\mu \mathrm{g}$ of RNA from each sample was then reverse transcribed into cDNA using the High capacity cDNA Reverse Transcription kit (Applied Biosystems \#4368814) according to manufacturer's instructions. Real-time PCR was the performed using the specific primers detail in Table 1. Real-time PCR was performed on an ABI7500 or ABI7900HT (Applied Biosystems) fast realtime PCR instrument.

\section{Table 1}

\begin{tabular}{|l|l|l|}
\hline Gene & Forward Primer & Reverse Primer \\
\hline Gapdh & CGTCCCGTAGACAAAATGGT & TTGATGGCAACAATCTCCAC \\
\hline
\end{tabular}




\begin{tabular}{|l|l|l|}
\hline NIrp3 & TCACAACTCGCCCAAGGAGGAA & AAGAGACCACGGCAGAAGCTAG \\
\hline II1 $\beta$ & GATCCACACTCTCCAGCTGCA & CAACCAACAAGTGATATTCTCCATG \\
\hline Kc $a$ & CATCTTCTCAAAATTCGAGTGACAA & TGGGAGTAGACAAGGTACAACCC \\
\hline Ifn $\beta$ & GCATTGAGCTGCGCTGTCAGTG & CTTGGGGACACCTTTTAGCATC \\
\hline Abcb1a & AACTCAGAGCCGCTTCTTCC & CTGTCCAGCCAACCTGCATA \\
\hline Abcb1b & GGTGGTGTCATTGTGGAGCAAG & GCATCAGTGTCACTCTGGGATC \\
\hline Abcg2 & CAGTTCTCAGCAGCTCTTCGAC & TCCTCCAGAGATGCCACGGATA \\
\hline Lpiat & TGCTATGGCACAGACTTCTGCG & ACTCAACAGCATGGTCCAGGCA \\
\hline Lclat1 & CCAGAAGGAACTGACCTCACAG & TCTCTTAGGCGGTCCACCACAA \\
\hline Cdipt & GGACTCCTAGACGCTTTCGATG & CAGGTTGACCAAGAGACACATGG \\
\hline
\end{tabular}

\section{Rhodamine 123 accumulation assay}

BMDMs or $A b c b 1 b^{-/-}$clones were seeded into 96-well plates at a concentration of $1 \times 10^{5}$ cells per well. Where indicated, BMDMs were treated with elacridar overnight $(2-10 \mu \mathrm{M})$. Rhodamine123 ( $2 \mu \mathrm{M}$,Sigma) was added and cells were incubated at $37^{\circ} \mathrm{C}$ for 30 minutes. Cells then washed with PBS 3 times to remove any extracellular rhodamine and DMEM media was replaced, after which cells were incubated for a further 30 minutes at $37^{\circ} \mathrm{C}$. Cells were then washed again with PBS and lysed in cell lysis buffer before being read at excitation and emission wavelengths of 485 and $535 \mathrm{~nm}$, respectively in a fluorescent plate reader.

\section{Total cholesterol measurement}

Total cholesterol was measured using Cholesterol Amplex Assay kit according to manufacturer's recommendations.

\section{Filipin staining}


WT and $A b c b 1 b^{-/}$cells were seeded onto coverslips at a concentration of $4 \times 10^{6}$ cells per well. After they had adhered, cells were fixed in $4 \%$ paraformaldehyde for 1 hour at room temperature. Cells were then stained with $25 \mu \mathrm{g} / \mathrm{ml}$ filipin (F9765, Sigma) overnight and washed 3 times with PBS before mounting on glass slides. Images were visualised on a Leica SP5 confocal microscope using $405 \mathrm{~nm}$ excitation and processed using Image J programme.

\section{GM1 staining}

Lipid rafts were assessed by specific labelling of endogenous GM1 ganglioside (a lipid raft marker) with the Vybrant Alexa Fluor 488 Lipid Raft labeling kit which makes use of fluorescently conjugated-CTB. The procedure was carried out according to manufacturer's instructions (Thermofisher). Samples were processed on the Attune NxT Flow Cytometer (Thermofisher) and analysed using FlowJo ${ }^{\mathrm{TM}}$ v10

\section{MALDI-MS Analysis}

WT and $A b c b 1 b^{-/-}$macrophages were cultured in DMEM before being trypsinised and analysed via mass spectrometry. Prior to analysis, the super-2,5-dihydroxybenzoic acid (Sigma-Aldrich, catalog no. 50862) matrix was added at a concentration of $10 \mathrm{mg} / \mathrm{mL}$ in a chloroform/methanol mixture at a $90: 10(\mathrm{v} / \mathrm{v})$ ratio; $0.4 \mu \mathrm{L}$ of a cell solution at a concentration of $2 \times 10^{5}$ to $2 \times 10^{6} \mathrm{~mL}^{-1}$, preliminary washed three times with double distilled water. corresponding to $\sim 100-1000$ cells per well of the MALDI target plate (384 Opti-TOF $123 \mathrm{~mm}$ $\times 84 \mathrm{~mm}$ AB Sciex NC0318050, 1016629), and $0.8 \mu \mathrm{L}$ of the matrix solution were deposited on the MALDI target plate. These were then gently mixed with a micropipette, and left to dry. MALDI-TOF MS analysis were performed on a 4800 Proteomics Analyzer (with TOF-TOF Optics, Applied Biosystems) using the reflectron mode. Samples were analyzed in the negative ion mode operating at $20 \mathrm{kV}$. A total of three independent experiments were performed. Data obtained from mass-spec were analyzed using Data Explorer version 4.9 (Applied Biosystems), and assignments were based on the MS/MS fragmentation profile.

\section{Statistical Analysis}


GraphPad Prism 7.0 software was used for data analysis. Data are represented as mean \pm SD and are representative of experiments done at least three times. Statistical significance was determined by unpaired Student's $t$ test; $p<0.05$ was considered statistically significant.

\section{Acknowledgements}

This work was supported in part by grants from The Wellcome Trust (108248/Z/15/Z), The Medical Research Council, UK (MR/S00968X/1) and core funds from Imperial College London to P.K.A.

The authors declare no competing financial interests.

\section{References}

1. H. Watson, Biological membranes. Essays Biochem. 59, 43-70 (2015).

2. H. E. Grecco, M. Schmick, P. I. H. Bastiaens, Signaling from the living plasma membrane. Cell 144, 897-909 (2011).

3. Y. Shimizu, The choreography of signaling at the plasma membrane. Immunol. Today 21, 413 (2000).

4. K. C. Barnett, J. C. Kagan, Lipids that directly regulate innate immune signal transduction. Innate Immun., 175342591985269 (2019).

5. R. Harkewicz, E. A. Dennis, Applications of Mass Spectrometry to Lipids and Membranes. Annu. Rev. Biochem. 80, 301-325 (2011).

6. M. S. Köberlin, et al., A Conserved Circular Network of Coregulated Lipids Modulates Innate Immune Responses. Cell 162, 170-183 (2015).

7. Ü. Coskun, K. Simons, Cell membranes: The lipid perspective. Structure 19, 15431548 (2011).

8. A. Z. Fernandis, M. R. Wenk, Membrane lipids as signaling molecules. Curr. Opin. Lipidol. 18, 121-128 (2007).

9. K. M. Eyster, The membrane and lipids as integral participants in signal transduction: Lipid signal transduction for the non-lipid biochemist. Am. J. Physiol. - Adv. Physiol. Educ. 31, 5-16 (2007). 
10. K. Simons, D. Toomre, Lipid rafts and signal transduction. Nat. Rev. Mol. Cell Biol. 1, $31-39(2000)$.

11. M. Triantafilou, K. Miyake, D. T. Golenbock, K. Triantafilou, Mediators of innate immune recognition of bacteria concentrate in lipid rafts and facilitate lipopolysaccharide-induced cell activation. J. Cell Sci. 115, 2603-2611 (2002).

12. J. C. Kagan, R. Medzhitov, Phosphoinositide-Mediated Adaptor Recruitment Controls Toll-like Receptor Signaling. Cell 125, 943-955 (2006).

13. A. Mathur, J. A. Hayward, S. M. Man, Molecular mechanisms of inflammasome signaling. J. Leukoc. Biol. 103, jlb.3MR0617-250R (2017).

14. H. Guo, J. B. Callaway, J. P.-Y. Ting, Inflammasomes: mechanism of action, role in disease, and therapeutics. Nat. Med. 21, 677-687 (2015).

15. Z. Zhong, et al., New mitochondrial DNA synthesis enables NLRP3 inflammasome activation. Nature (2018) https:/doi.org/10.1038/s41586-018-0372-z.

16. F. G. Bauernfeind, et al., Cutting edge: NF-kappaB activating pattern recognition and cytokine receptors license NLRP3 inflammasome activation by regulating NLRP3 expression. J. Immunol. 183, 787-91 (2009).

17. C. Hamilton, L. Tan, T. Miethke, P. K. Anand, Immunity to uropathogens: The emerging roles of inflammasomes. Nat. Rev. Urol. 14, 284-295 (2017).

18. C. R. Lupfer, M. D. Rippee-Brooks, P. K. Anand, "Common Differences: The Ability of Inflammasomes to Distinguish Between Self and Pathogen Nucleic Acids During Infection" in International Review of Cell and Molecular Biology, (2019), pp. 139-172.

19. C. Lupfer, P. K. Anand, Integrating Inflammasome Signaling in Sexually Transmitted Infections. Trends Immunol. 37, 703-714 (2016).

20. R. G. Carroll, et al., An unexpected link between fatty acid synthase and cholesterol synthesis in proinflammatory macrophage activation. J. Biol. Chem. 293, 5509-5521 (2018).

21. M. S. Köberlin, L. X. Heinz, G. Superti-Furga, Functional crosstalk between membrane lipids and TLR biology. Curr. Opin. Cell Biol. 39, 28-36 (2016).

22. J. M. Ruysschaert, C. Lonez, Role of lipid microdomains in TLR-mediated signalling. Biochim. Biophys. Acta - Biomembr. 1848, 1860-1867 (2015).

23. A. T. Shamshiev, et al., Dyslipidemia inhibits Toll-like receptor-induced activation of 
CD8a-negative dendritic cells and protective Th1 type immunity. J. Exp. Med. (2007) https:/doi.org/10.1084/jem.20061737.

24. A. G. York, et al., Limiting Cholesterol Biosynthetic Flux Spontaneously Engages Type i IFN Signaling. Cell 163, 1716-1729 (2015).

25. M. de la Roche, et al., Trafficking of cholesterol to the ER is required for NLRP3 inflammasome activation. J. Cell Biol., jcb.201709057 (2018).

26. E. J. Tarling, T. Q. d. A. Vallim, P. A. Edwards, Role of ABC transporters in lipid transport and human disease. Trends Endocrinol. Metab. 24, 342-350 (2013).

27. C. L. Jackson, L. Walch, J. M. Verbavatz, Lipids and Their Trafficking: An Integral Part of Cellular Organization. Dev. Cell 39, 139-153 (2016).

28. E. Ikonen, Cellular cholesterol trafficking and compartmentalization. Nat. Rev. Mol. Cell Biol. 9, 125-138 (2008).

29. E. Ikonen, Mechanisms of cellular cholesterol compartmentalization: recent insights. Curr. Opin. Cell Biol. 53, 77-83 (2018).

30. N. Wang, D. Lan, W. Chen, F. Matsuura, A. R. Tall, ATP-binding cassette transporters G1 and G4 mediate cellular cholesterol efflux to high-density lipoproteins. Proc. Natl. Acad. Sci. (2004) https:/doi.org/10.1073/pnas.0403506101.

31. L. Yvan-Charvet, et al., Combined deficiency of ABCA1 and ABCG1 promotes foam cell accumulation and accelerates atherosclerosis in mice. J. Clin. Invest. 117, 39003908 (2007).

32. L. M. Hodges, et al., Very important pharmacogene summary: ABCB1 (MDR1, Pglycoprotein). Pharmacogenet. Genomics (2011) https:/doi.org/10.1097/FPC.0b013e3283385a1c.

33. G. Van Meer, D. Halter, H. Sprong, P. Somerharju, M. R. Egmond, ABC lipid transporters: Extruders, flippases, or flopless activators? FEBS Lett. 580, 1171-1177 (2006).

34. Y. Romsicki, F. J. Sharom, Phospholipid flippase activity of the reconstituted Pglycoprotein multidrug transporter. Biochemistry 40, 6937-6947 (2001).

35. M. Bentires-Alj, et al., NF-kappaB transcription factor induces drug resistance through MDR1 expression in cancer cells. Oncogene 22, 90-97 (2003).

36. J. Liu, et al., Chronic inflammation up-regulates P-gp in peripheral mononuclear blood 
cells via the STAT3/Nf-kb pathway in 2,4,6-trinitrobenzene sulfonic acid-induced colitis mice. Sci. Rep. (2015) https:/doi.org/10.1038/srep13558.

37. F. Hyafil, C. Vergely, P. Du Vignaud, T. Grand-Perret, In Vitro and in Vivo Reversal of Multidrug Resistance by GF120918, an Acridonecarboxamide Derivative. Cancer Res. 53, 4595-4602 (1993).

38. R. P. Dash, R. Jayachandra Babu, N. R. Srinivas, Therapeutic Potential and Utility of Elacridar with Respect to P-glycoprotein Inhibition: An Insight from the Published In Vitro, Preclinical and Clinical Studies. Eur. J. Drug Metab. Pharmacokinet. (2017) https:/doi.org/10.1007/s13318-017-0411-4.

39. S. Forster, A. E. Thumser, S. R. Hood, N. Plant, Characterization of rhodamine-123 as a tracer dye for use in in vitro drug transport assays. PLoS One (2012) https:/doi.org/10.1371/journal.pone.0033253.

40. C. Hamilton, P. K. Anand, Right place, right time: localisation and assembly of the NLRP3 inflammasome. F1000Research (2019) https:/doi.org/10.12688/f1000research.18557.1.

41. T. Kawasaki, T. Kawai, Toll-like receptor signaling pathways. Front. Immunol. 5, 461 (2014).

42. K. Simons, R. Ehehalt, Cholesterol, lipid rafts, and disease. J. Clin. Invest. 110, 597603 (2002).

43. I. Levental, M. Grzybek, K. Simons, Greasing their way: Lipid modifications determine protein association with membrane rafts. Biochemistry 49, 6305-6316 (2010).

44. S. Munro, Lipid Rafts: Elusive or Illusive? Cell 115, 377-388 (2003).

45. A. Garrigues, A. E. Escargueil, S. Orlowski, The multidrug transporter, P-glycoprotein, actively mediates cholesterol redistribution in the cell membrane. Proc Natl Acad Sci U S A 99, 10347-10352 (2002).

46. G. D. Luker, K. R. Nilsson, D. F. Covey, D. Piwnica-Worms, Multidrug resistance (MDR1) P-glycoprotein enhances esterification of plasma membrane cholesterol. J. Biol. Chem. (1999) https:/doi.org/10.1074/jbc.274.11.6979.

47. M. Aureli, et al., GM1 Ganglioside: Past Studies and Future Potential. Mol. Neurobiol. 53, 1824-1842 (2016).

48. Y. Sugimoto, et al., "Accumulation of cholera toxin and GM1 ganglioside in the early endosome of Niemann-Pick C1-deficient cells" (January 13, 2020). 
49. G. Van Meer, D. R. Voelker, G. W. Feigenson, Membrane lipids: Where they are and how they behave. Nat. Rev. Mol. Cell Biol. 9, 112-124 (2008).

50. N. J. Blunsom, S. Cockcroft, Phosphatidylinositol synthesis at the endoplasmic reticulum. Biochim. Biophys. acta. Mol. cell Biol. lipids 1865 (2020).

51. D. Barneda, S. Cosulich, L. Stephens, P. Hawkins, How is the acyl chain composition of phosphoinositides created and does it matter? Biochem. Soc. Trans. (2019) https:/doi.org/10.1042/BST20190205.

52. A. Naguib, et al., P53 mutations change phosphatidylinositol acyl chain composition. Cell Rep. 10, 8-19 (2015).

53. P. K. Anand, et al., TLR2 and RIP2 pathways mediate autophagy of Listeria monocytogenes via extracellular signal-regulated kinase (ERK) activation. J. Biol. Chem. 286, 42981-42991 (2011).

54. P. K. Anand, et al., NLRP6 negatively regulates innate immunity and host defence against bacterial pathogens. Nature 488 (2012). 
A

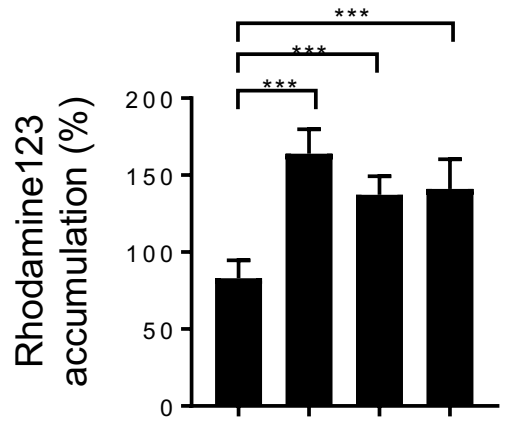

Elacridar

D

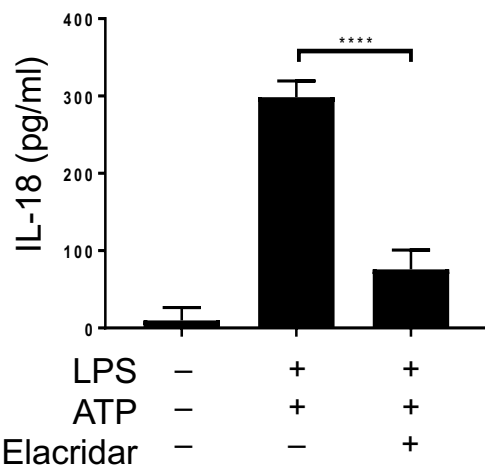

G

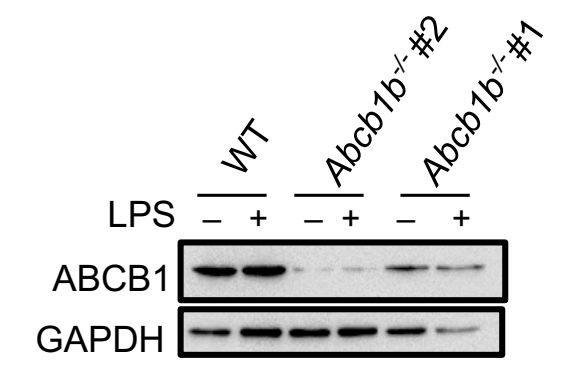

$\mathbf{J}$

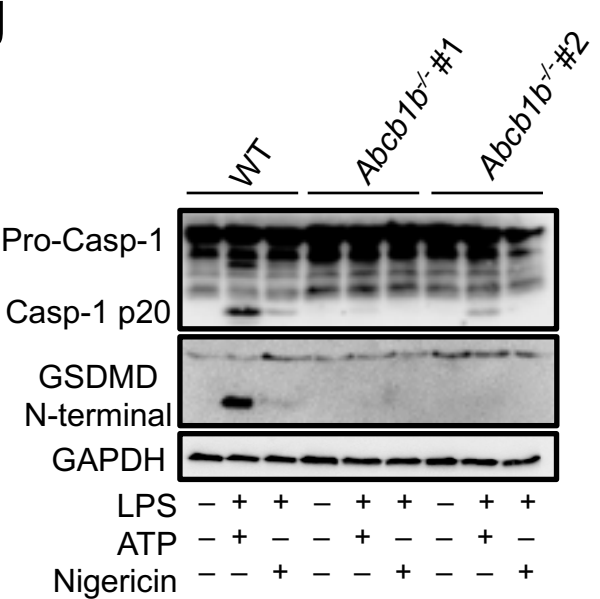

B

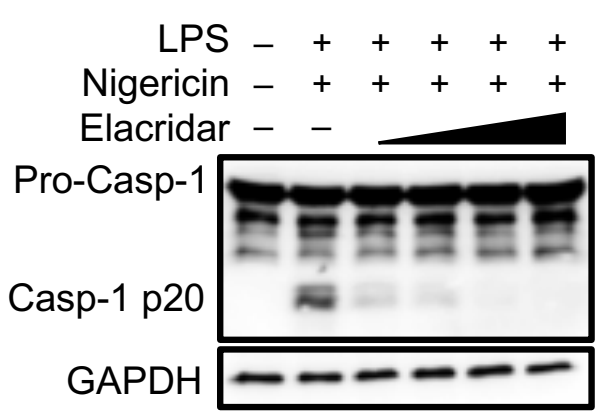

E

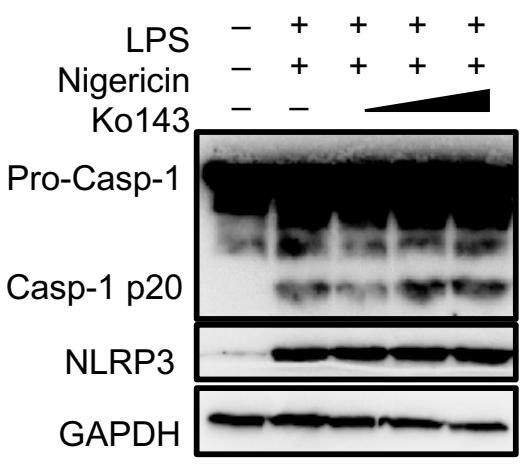

H
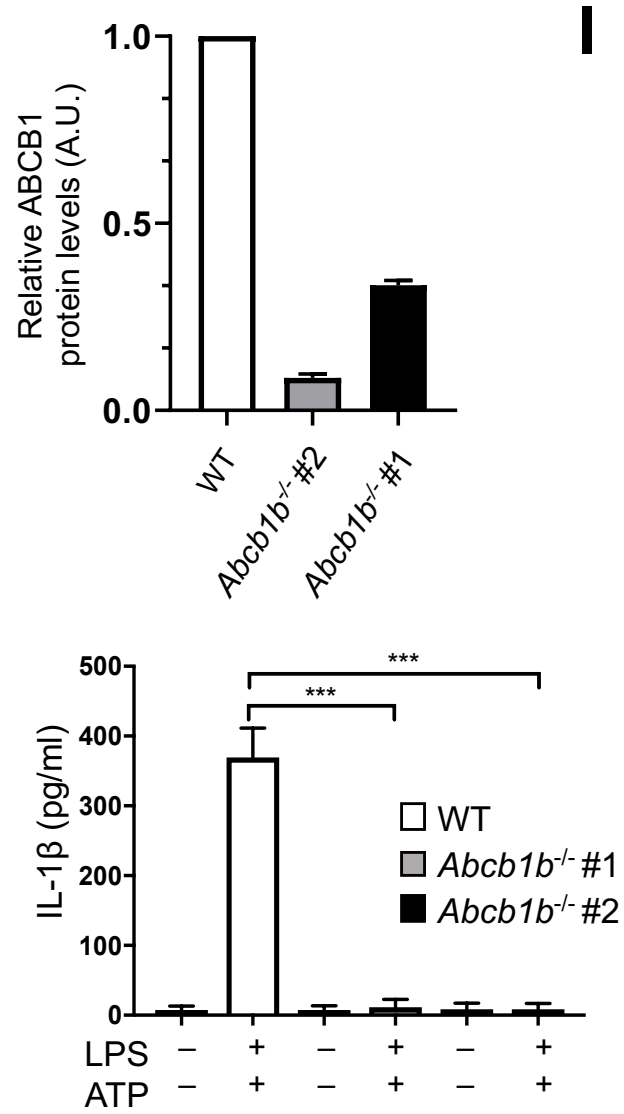

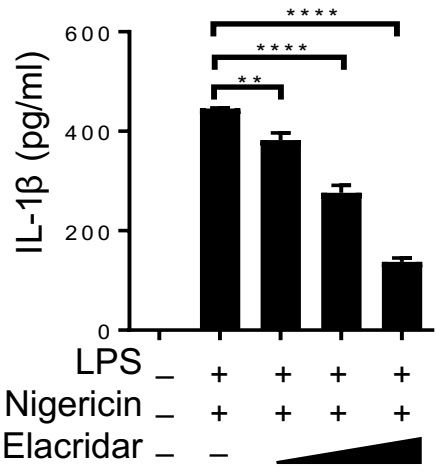

F
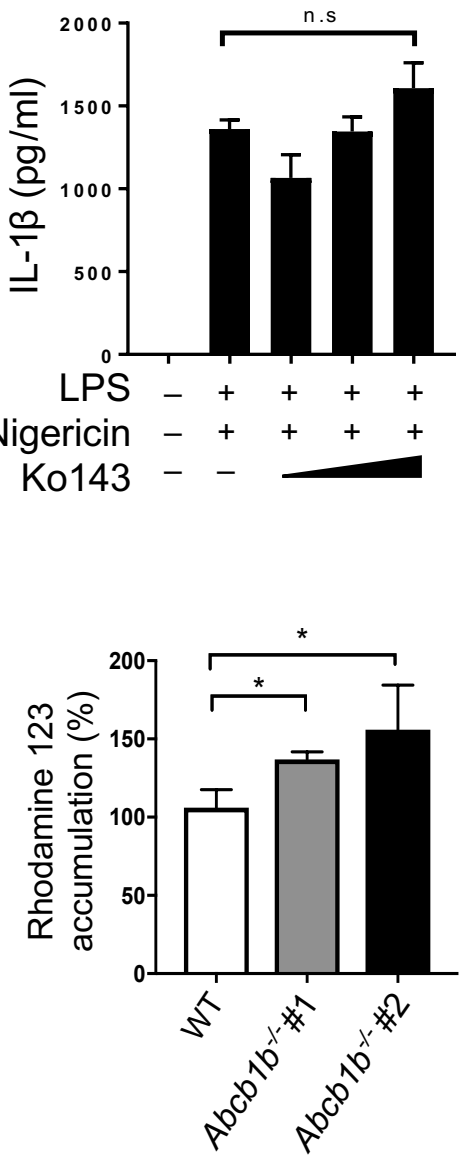
A

D

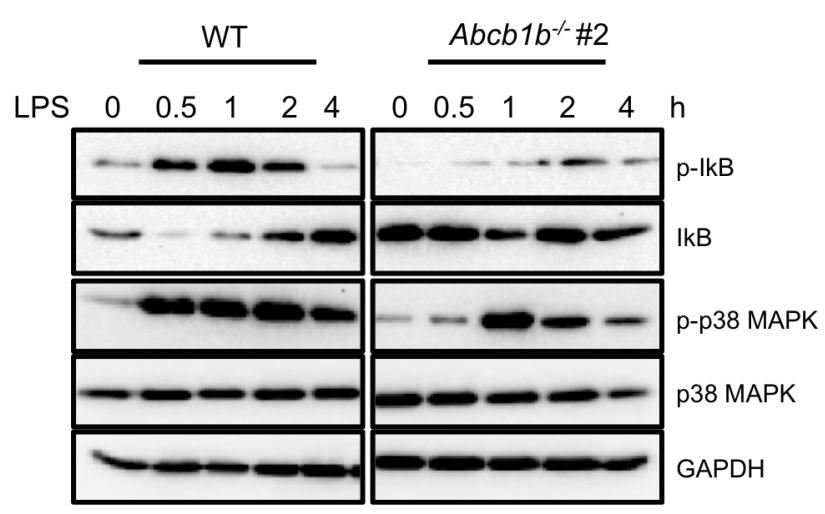

$\mathbf{F}$

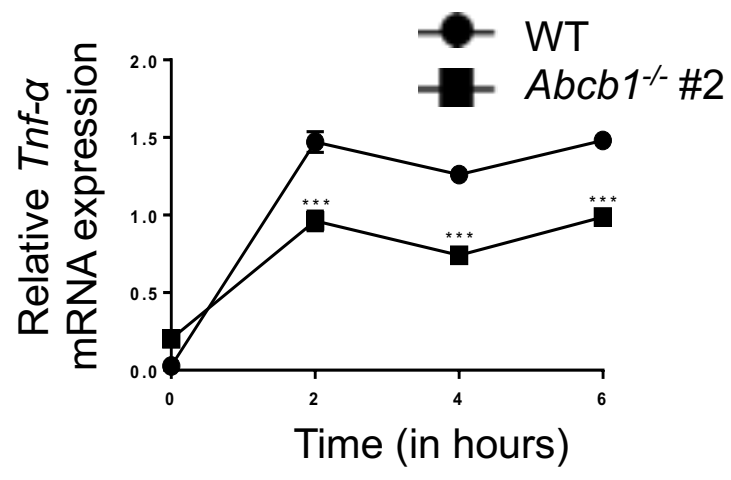

H

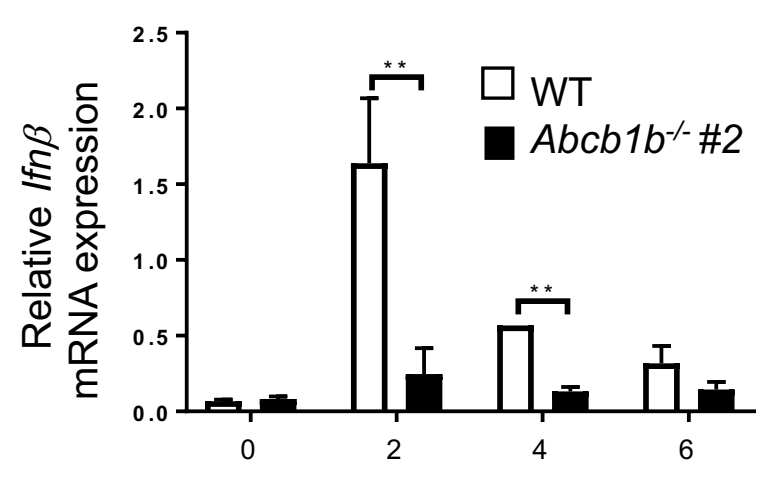

$\mathbf{E}$

WT

$A b c b 1^{1-\# 2}$

Time (in hours)

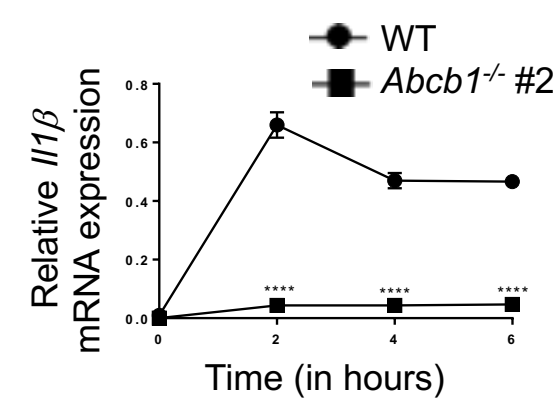

C

G
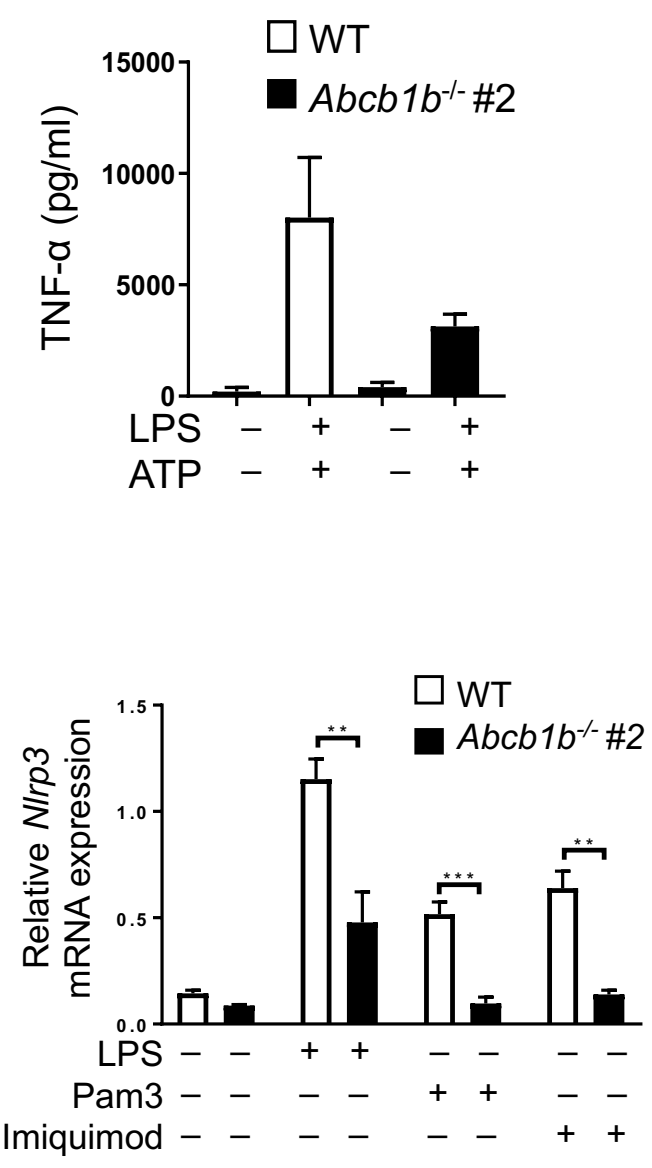

Figure 2 
A
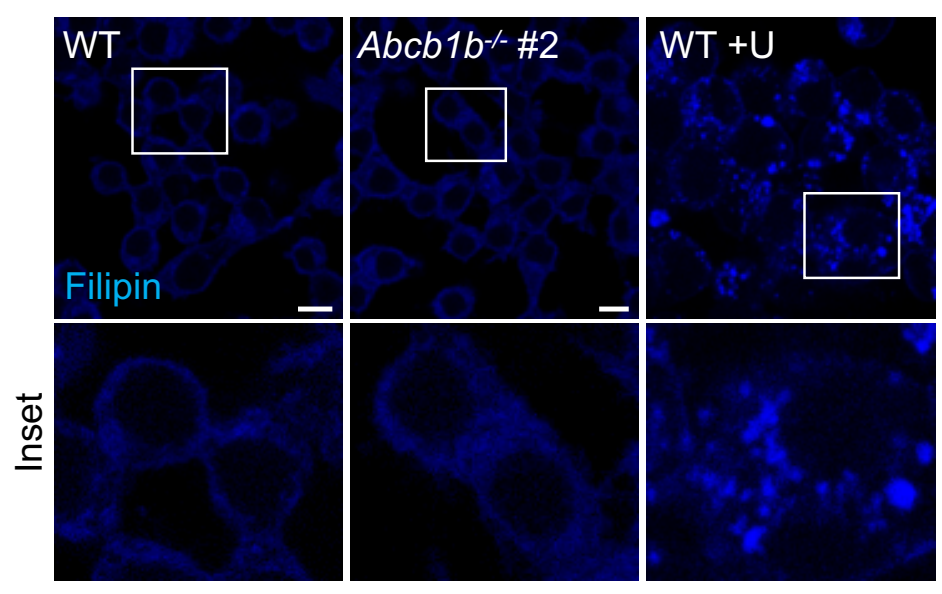

B

C

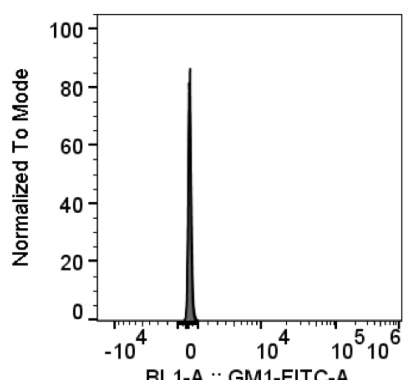

E
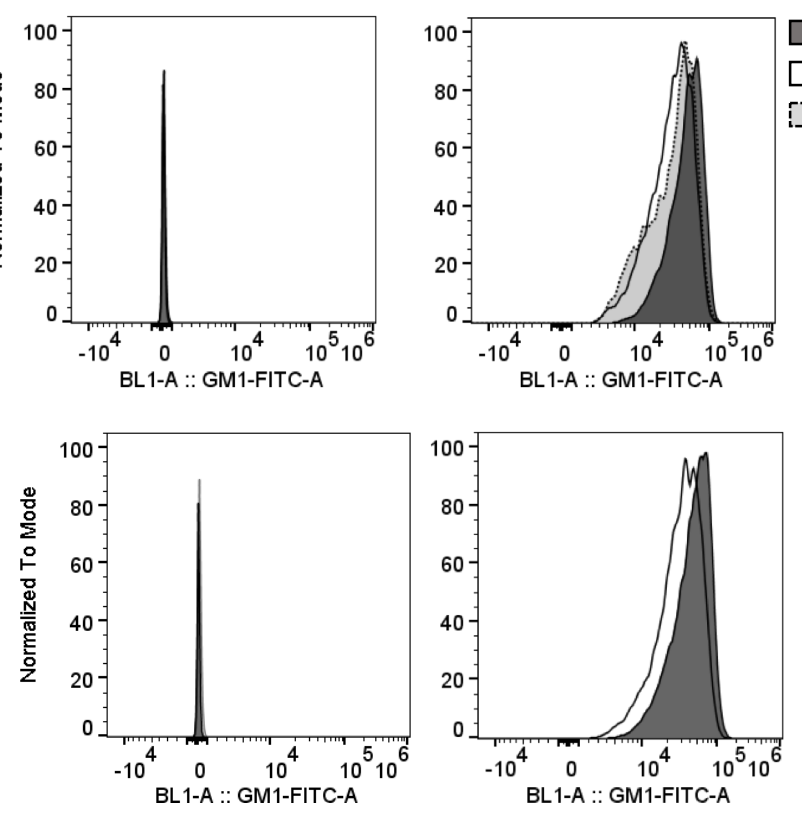

$\square$ WT

$\square A b c b 1 b^{-1-\# 2}$

G

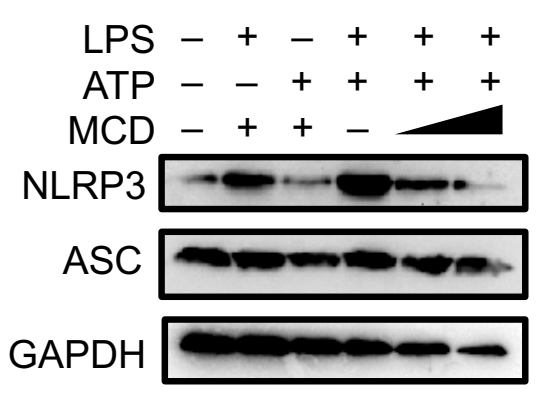

H
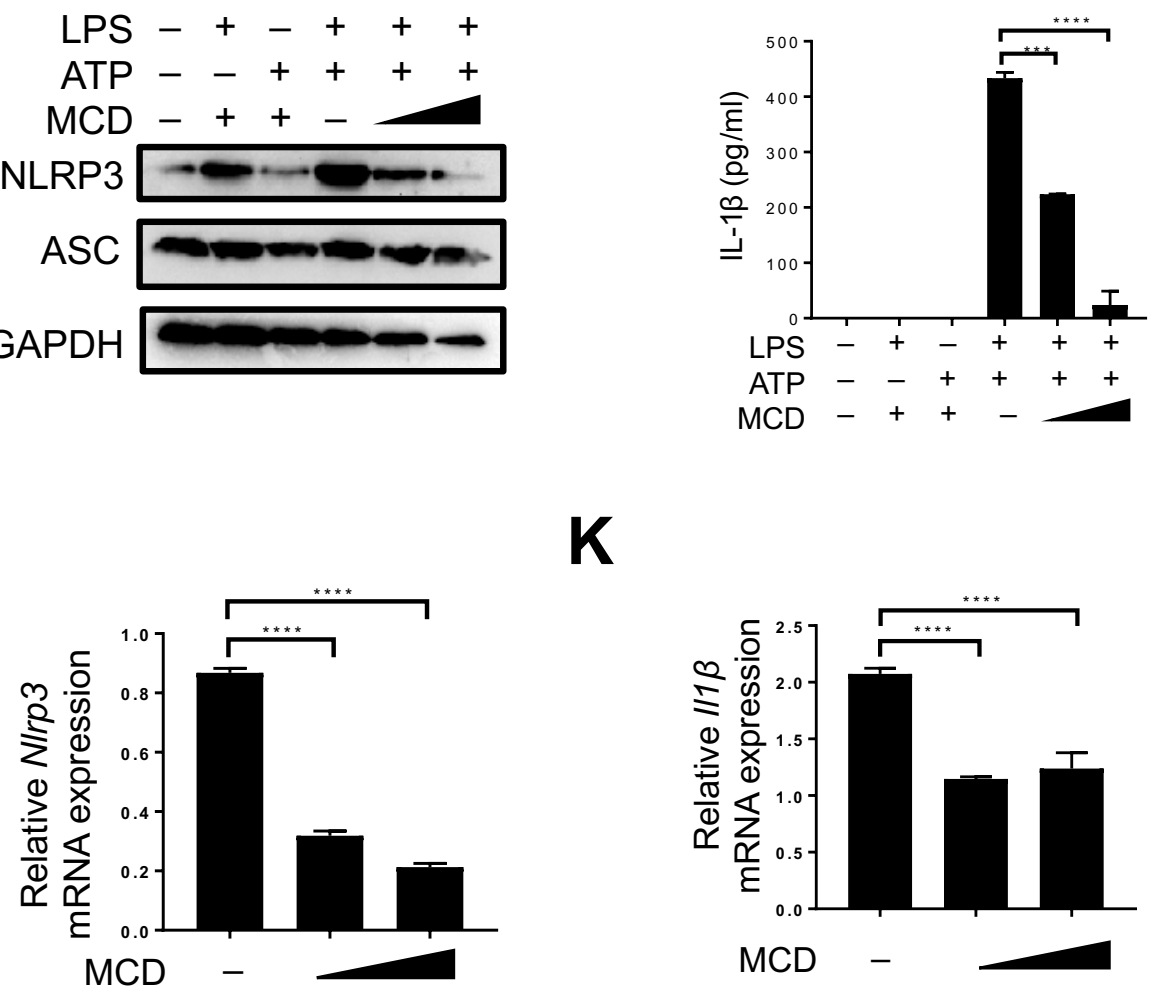

$\square$ WT

$\square A b c b 1 b^{-/-} \# 2$

MCD

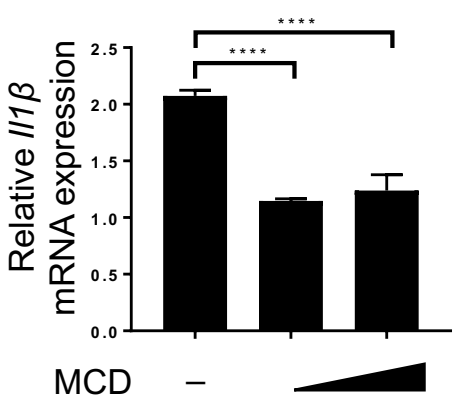

D

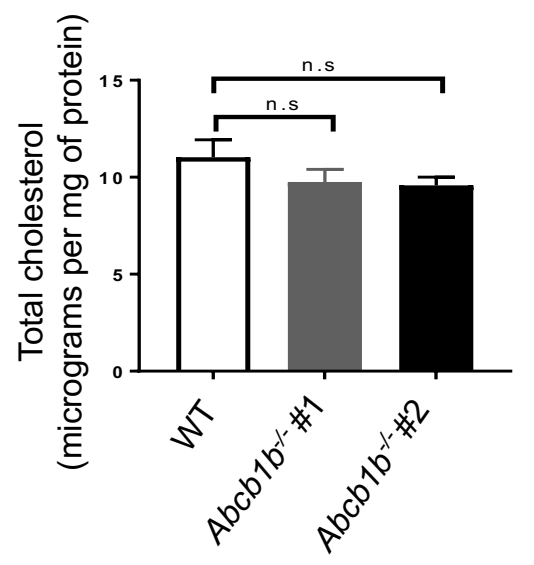

F
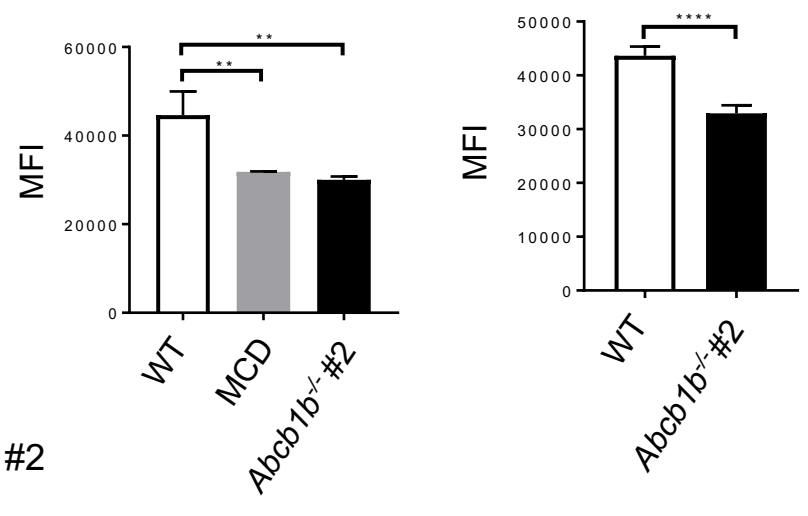

I
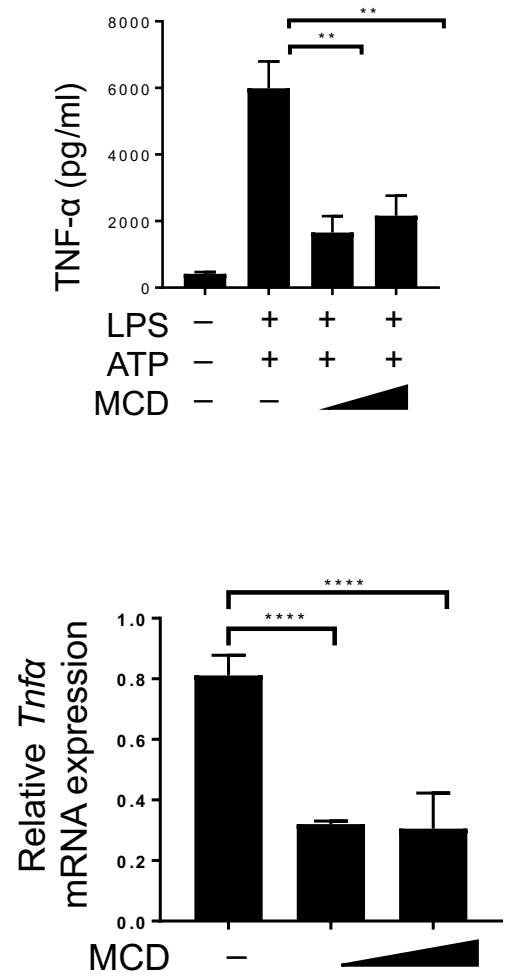

Figure 3 
A

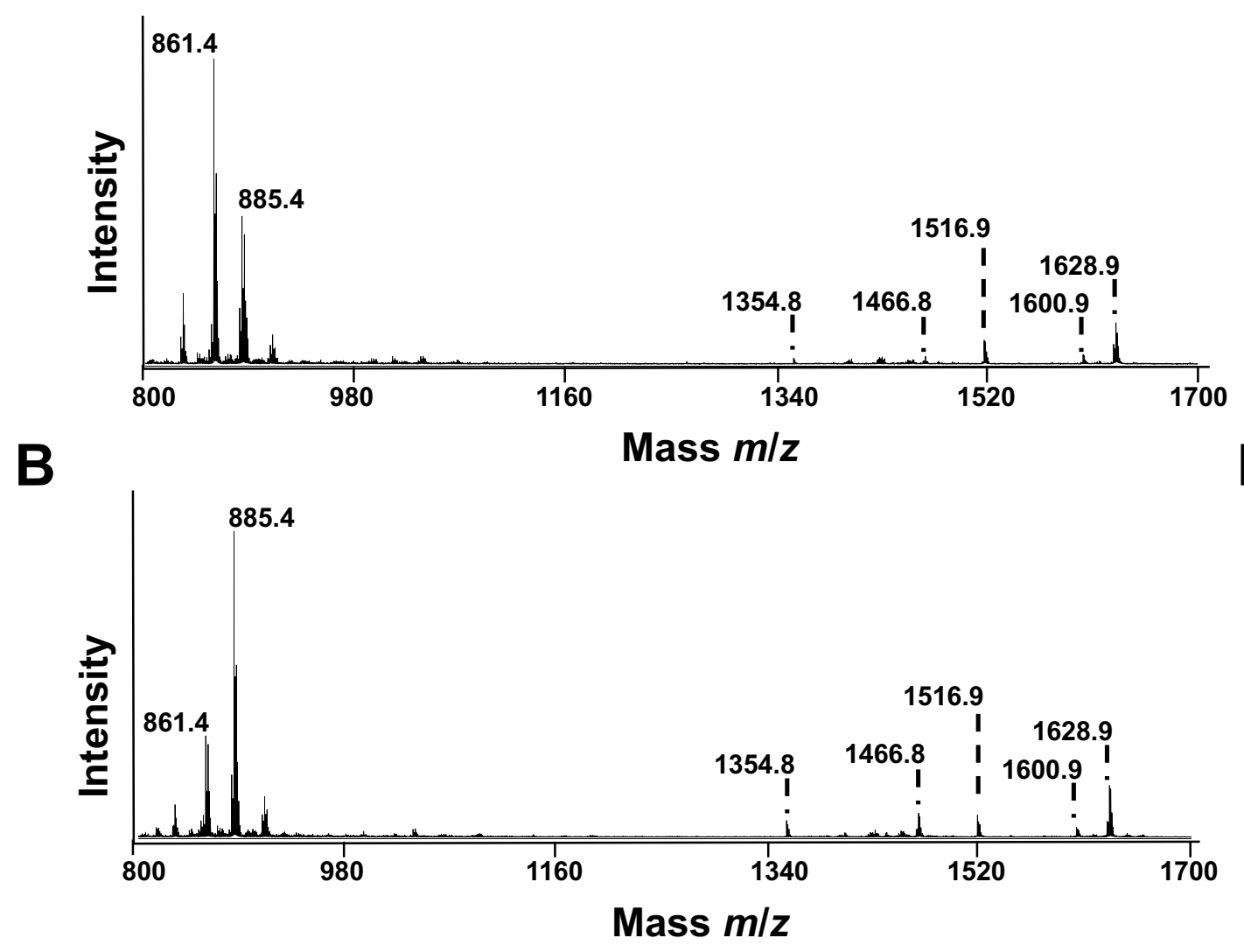

C

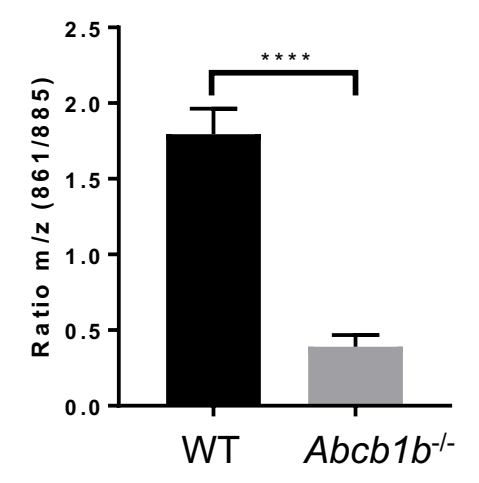

D

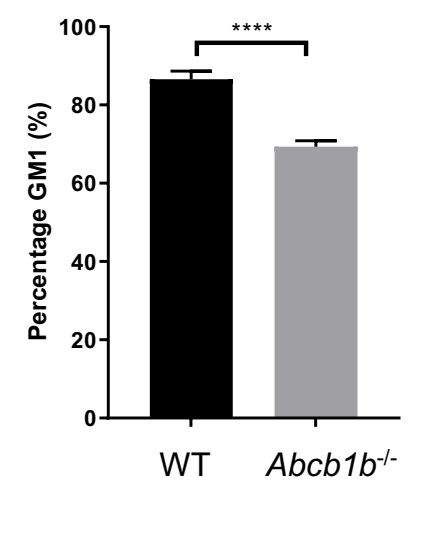

E F
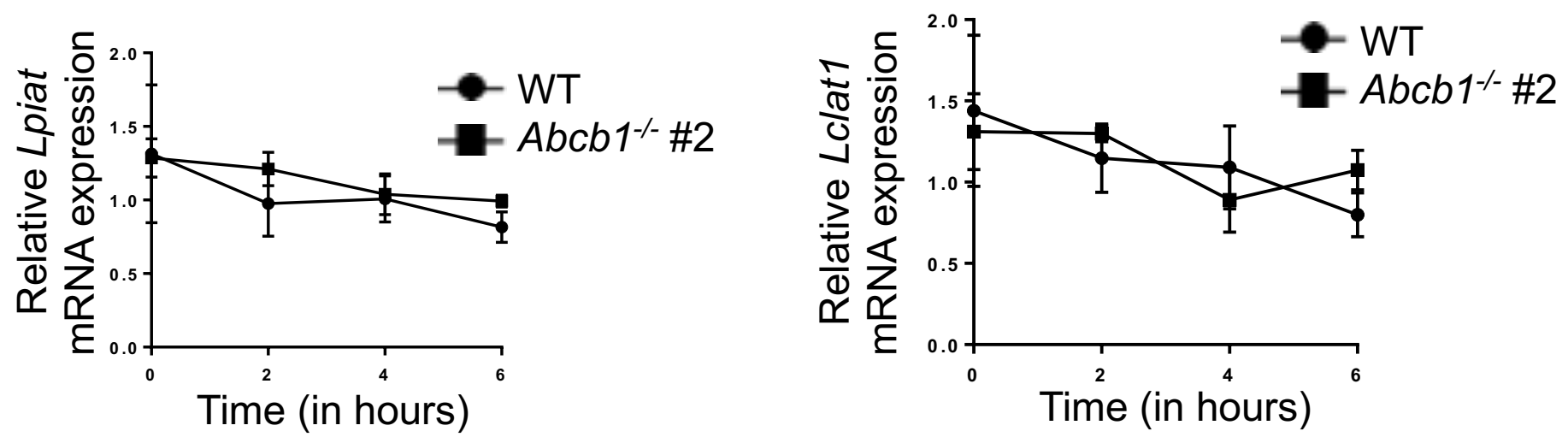

G

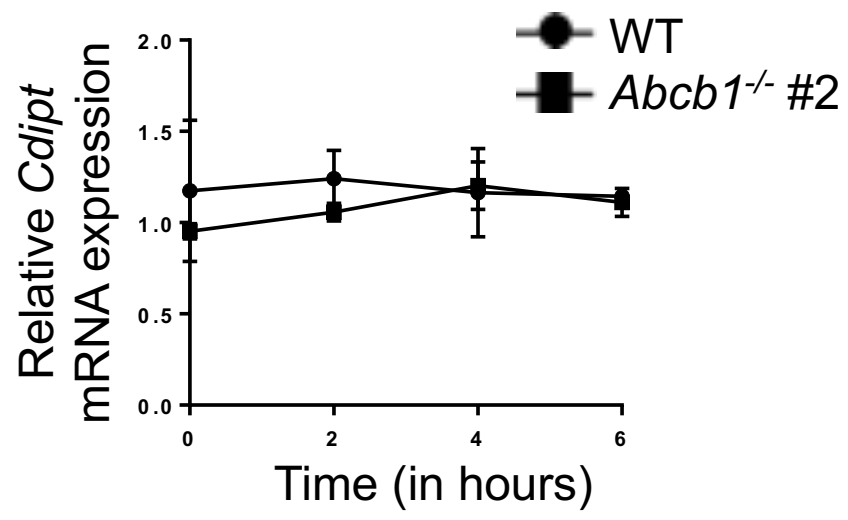


A

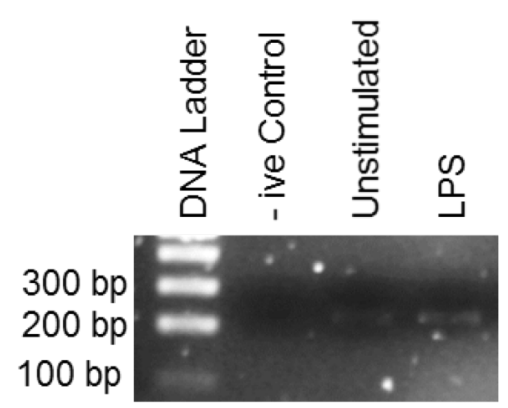

D

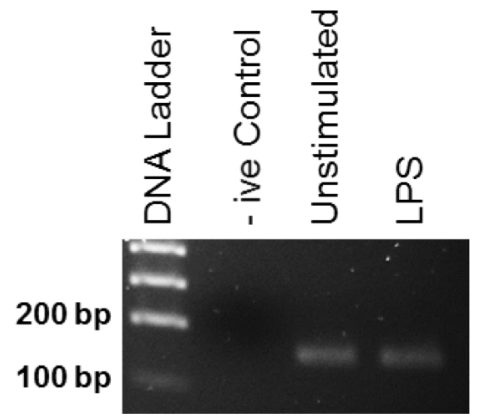

F

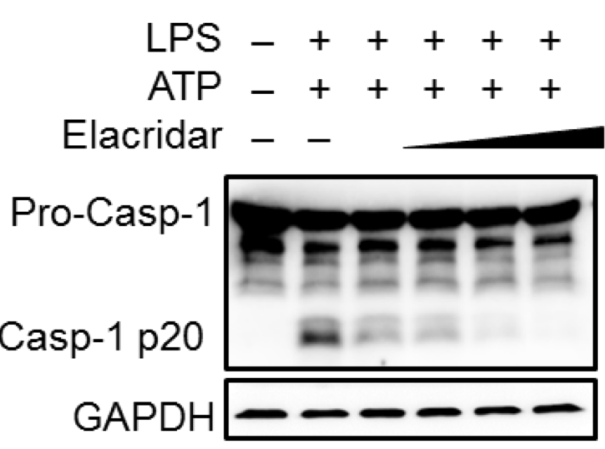

H

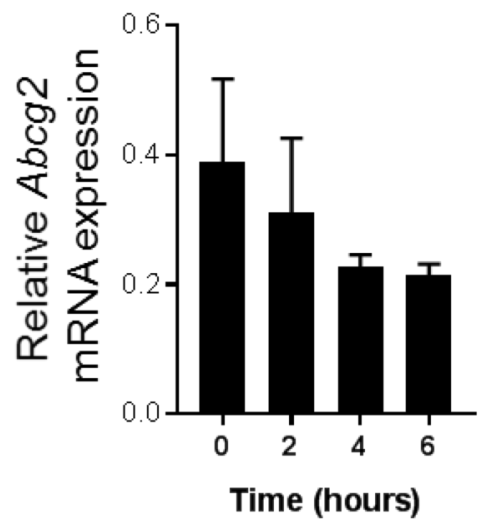

B
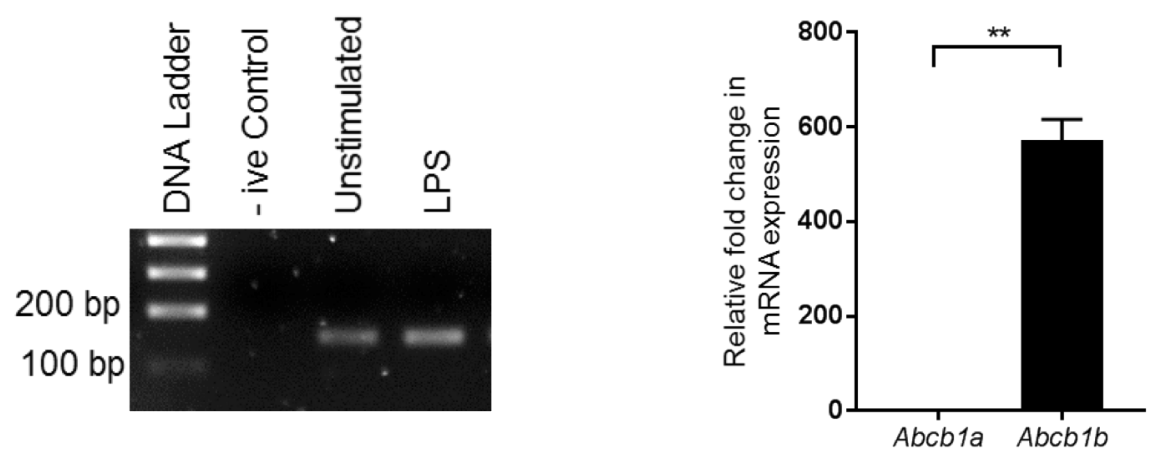

E

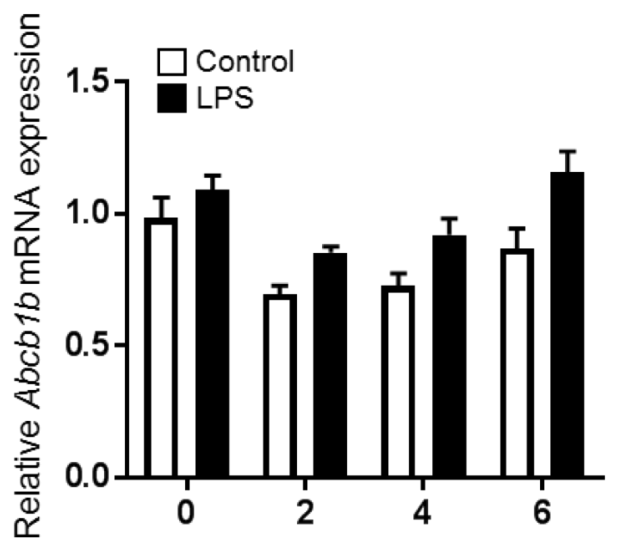

G

Time (hours)
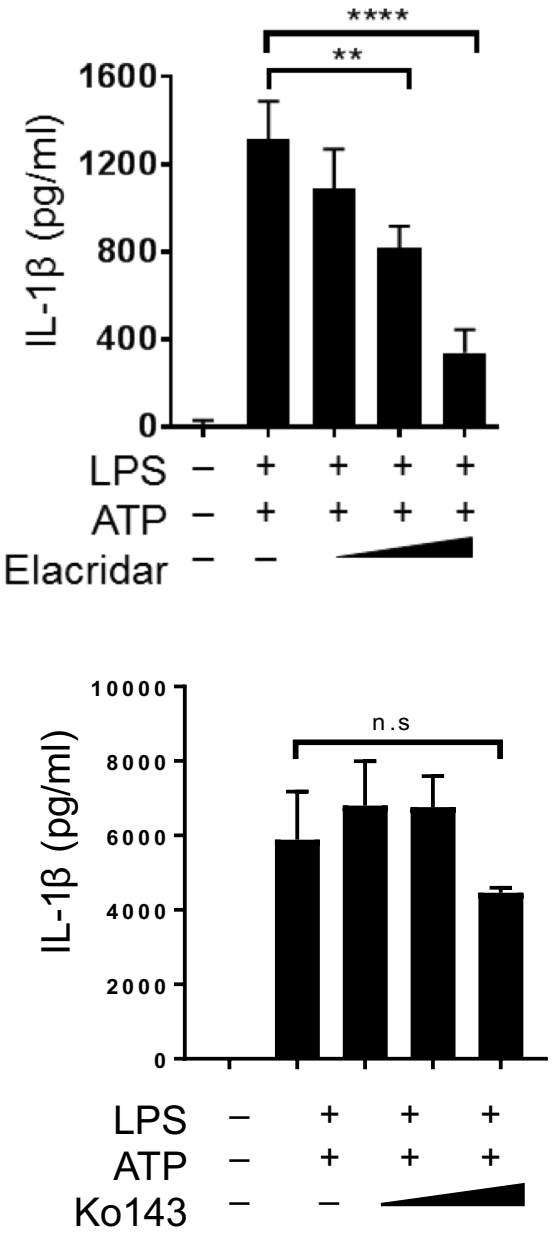


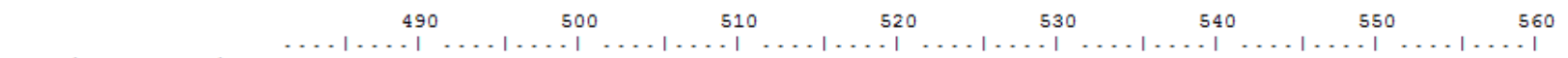
ABCB1+/+ Exon 10/11 GCTTCTCAAC AAAGGGCTAC AAACCAGACA GTATAATGGG AAACTTAGAG TTTAAAAATG TTCACTTCAA CTACCCATCG 556 ABCB1-/- \#1 GCTTCTCAAC AAAGGGTAC AAACCAGACA GTATAATGGG AAACTTATAG TGTATGGAGG TTCTATTCTT CTACCC---- 522 $\mathrm{ABCB} 1-/-\# 2$ GCTTCTCAAC AAAGGGCTAC AAACCAAACA GTATAATGGG AAACTTAGAG TTTAAAAATG TTCACTTCAA CTACCCATCG 529

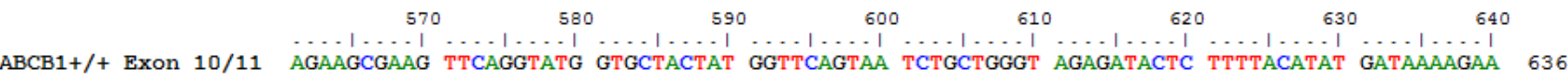
$\mathrm{ABCB} 1-/-\# 1$ TCTGCTGGGT AGAGATACTC TTTTACATAG GAGGGAAGA

ABCB1-/- \#2

AGAAGCGAA

TG GGGCTGGTGT GGCTATGGAT TCTGCTGCTG CTAGATACAC ATTTACTTAT GATTATGAAA 600

B

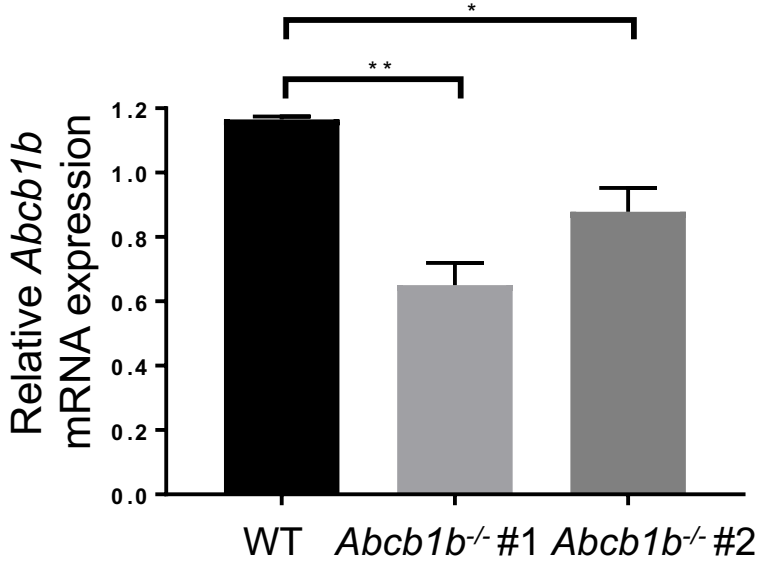


A

更

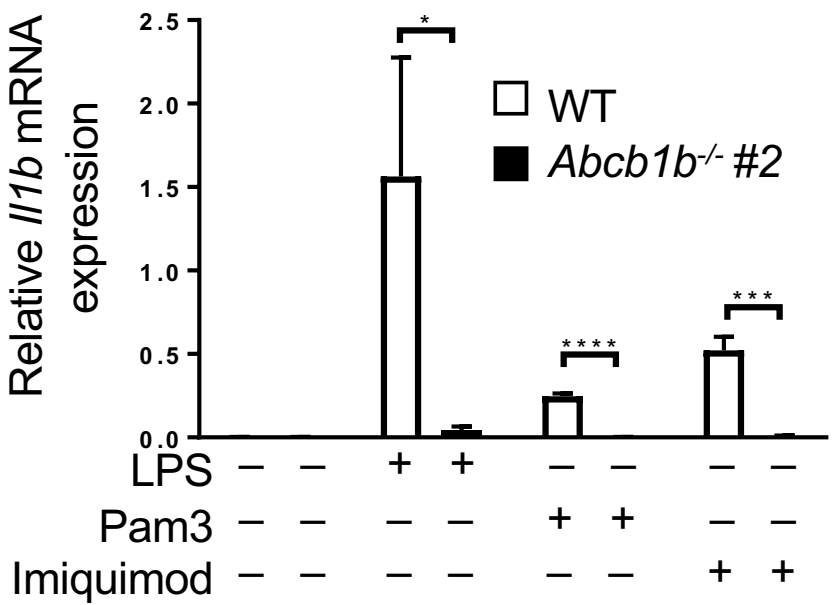

C

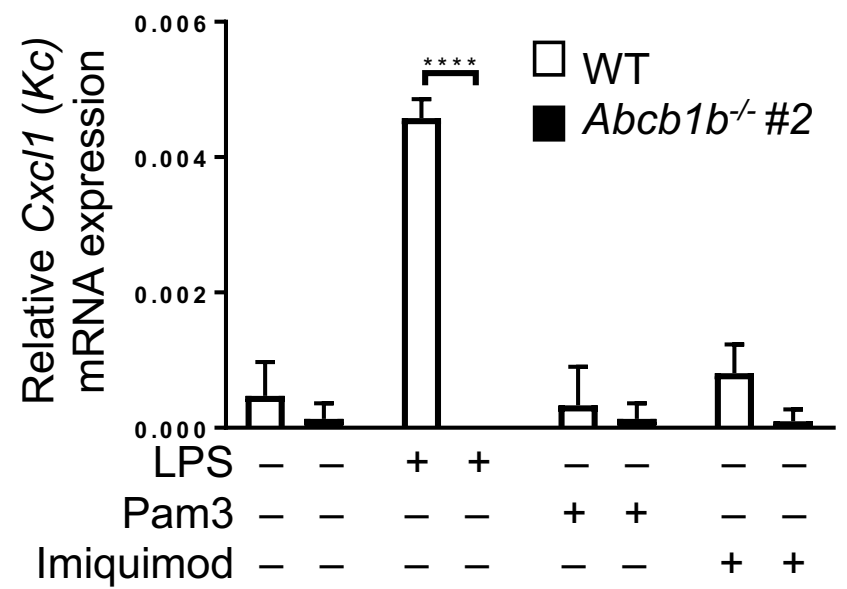

B

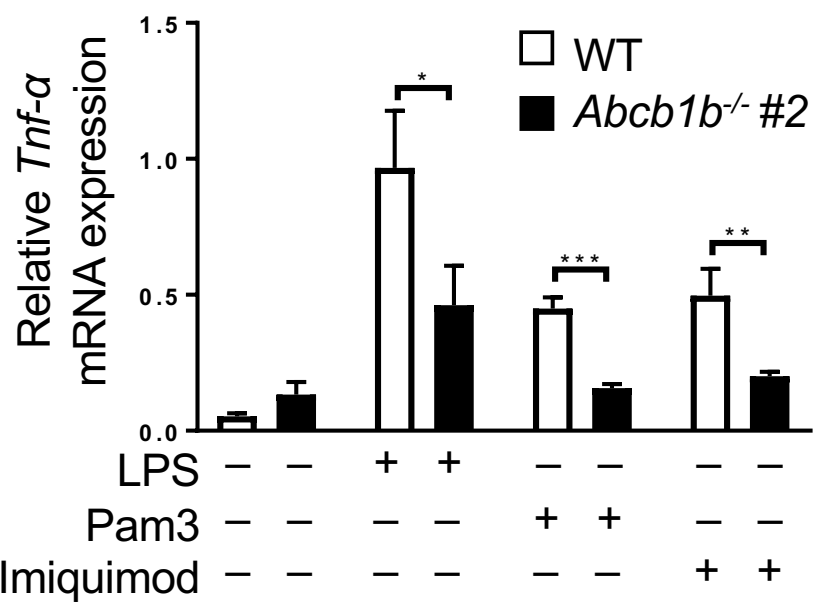


A

B
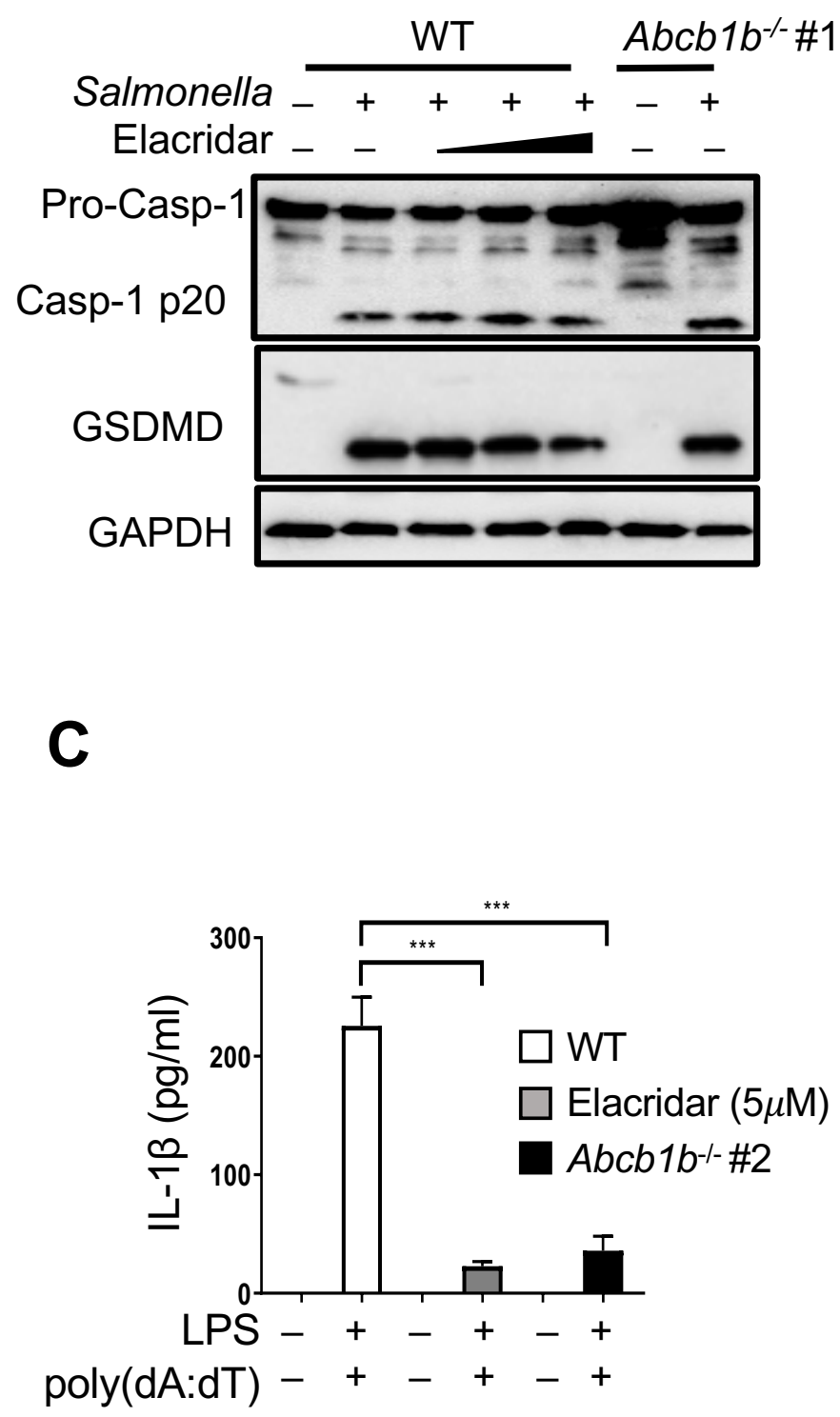

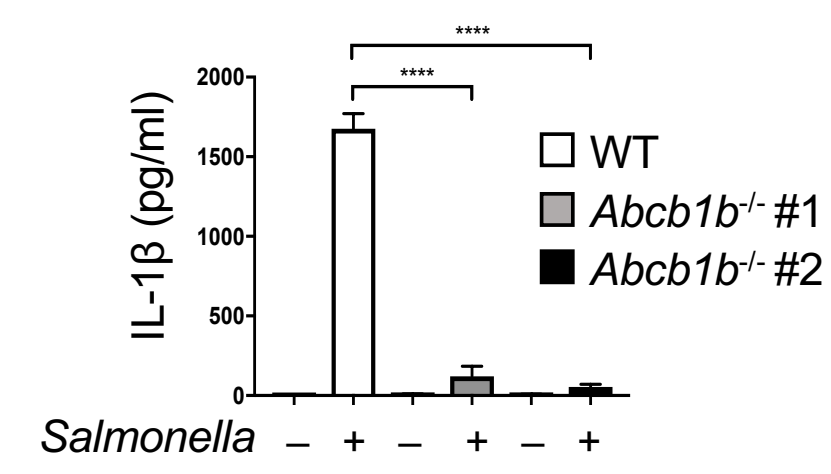

D

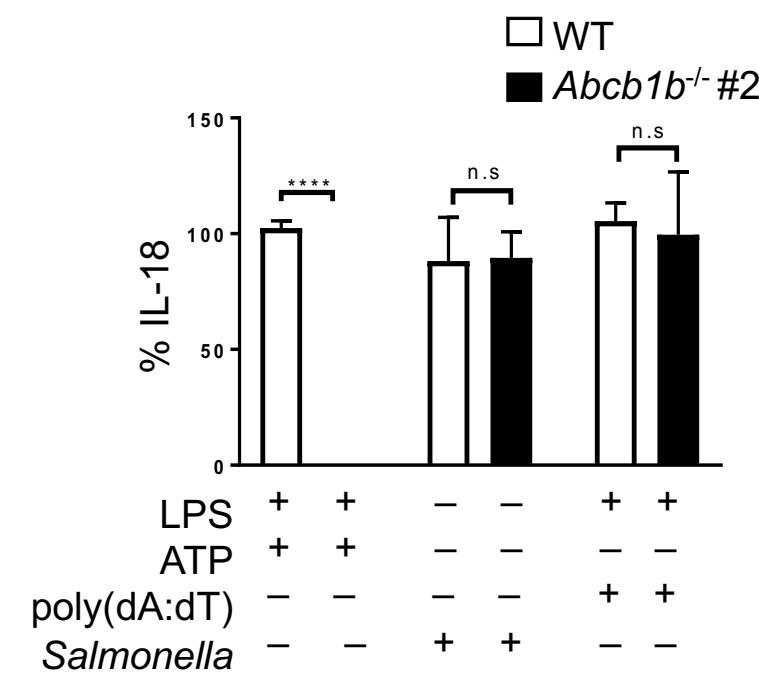


A

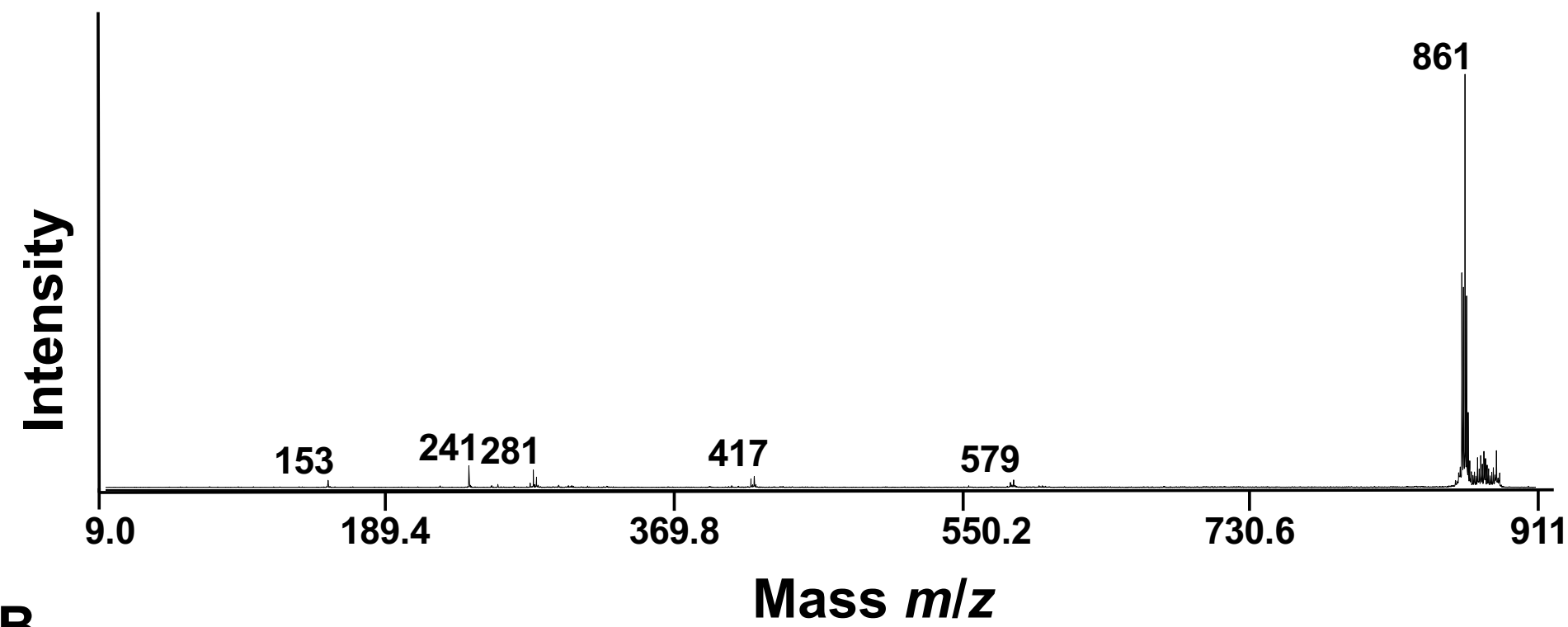

B

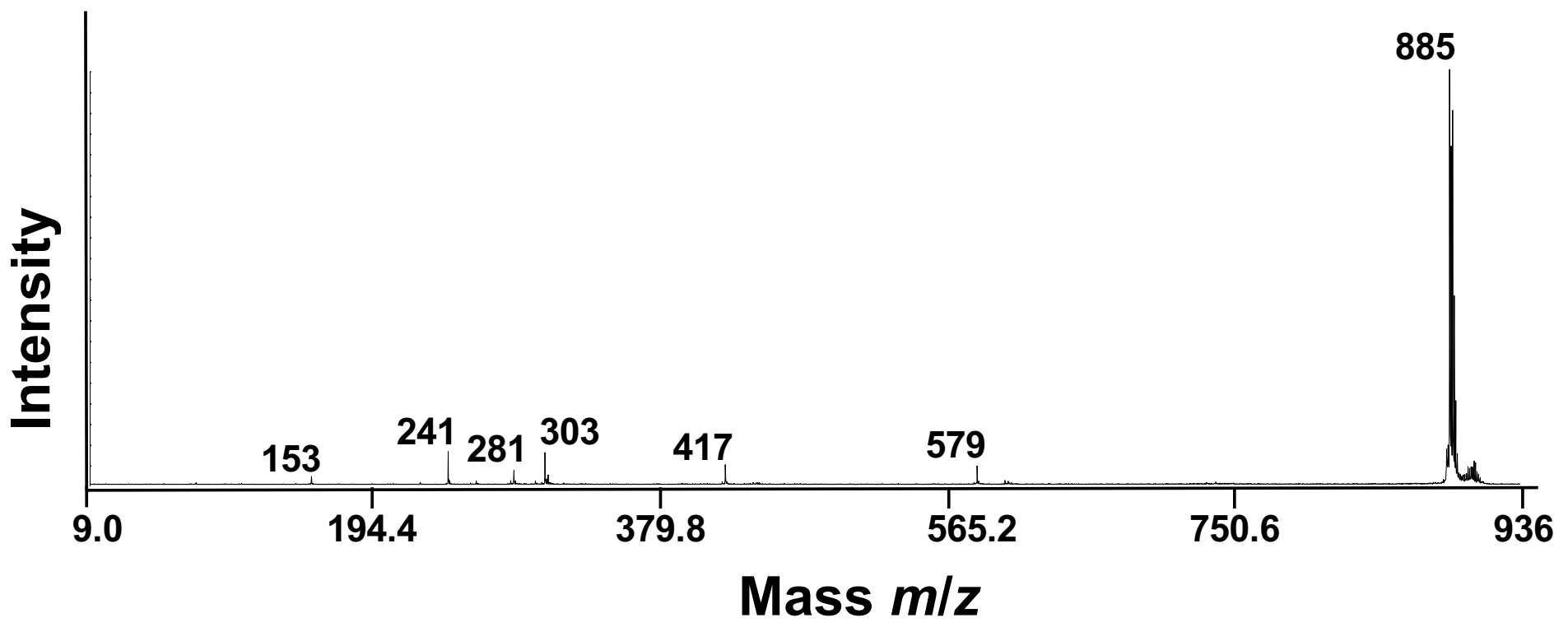

